\title{
The Inverse $s$-Wave Scattering Problem for a Class of Potentials Depending on Energy
}

\author{
M. Jaulent and C. Jean \\ Département de Physique Mathématique`, Montpellier, France
}

Received November 16, 1971

\begin{abstract}
The inverse scattering problem is considered for the radial $s$-wave Schrödinger equation with the energy-dependent potential $V^{+}(E, x)=U(x)+2 \sqrt{E} Q(x)$. (Note that this problem is closely related to the inverse problem for the radial $s$-wave KleinGordon equation of zero mass with a static potential.) Some authors have already studied it by extending the method given by Gel'fand and Levitan in the case $Q=0$. Here, a more direct approach generalizing the Marchenko method is used. First, the Jost solution $f^{+}(E, x)$ is shown to be generated by two functions $F^{+}(x)$ and $A^{+}(x, t)$. After introducing the potential $V^{-}(E, x)=U(x)-2 \sqrt{E} Q(x)$ and the corresponding functions $F^{-}(x)$ and $A^{-}(x, t)$, fundamental integral equations are derived connecting $F^{+}(x), F^{-}(x), A^{+}(x, t)$ and $A^{-}(x, t)$ with two functions $z^{+}(x)$ and $z^{-}(x) ; z^{+}(x)$ and $z^{-}(x)$ are themselves easily connected with the binding energies $E_{n}^{+}$and the scattering "matrix" $S^{+}(E), E>0$ (the input data of the inverse problem). The inverse problem is then reduced to the solution of these fundamental integral equations. Some specific examples are given. Derivation of more elaborate results in the case of real potentials, and applications of this work to other inverse problems in physics will be the object of further studies.
\end{abstract}

\section{Introduction}

The problem of describing the interactions between colliding particles is of fundamental interest in physics. In many cases, a description can be carried out through a well known theoretical model. To this end, the following "inverse problem" is investigated: having determined some important quantities from experimental results, what are the values of the parameters occurring in the chosen model which reproduce them?

In particular, we are interested in collisions of two spinless particles, and we suppose that the $s$-wave scattering "matrix" $S(E)$ (defined for all energies $E>0$ ) and the $s$-wave binding energies $E_{n}$ are exactly known from collision experiments.

We can try first to find a radial static potential $V(x)(x \geqq 0)$ which yields the given $S(E)(E>0)$ and $E_{n}$ through the radial $s$-wave Schrödinger equation. We recall that this equation is written as follows, in the center-of-mass of the two particles, and with the usual reduced variables:

$$
y^{\prime \prime}+[E-V(x)] y=0, \quad x \geqq 0 .
$$

* Physique Mathématique et Théorique, Equipe de recherche associée au C.N.R.S.

13 Commun. math Phys., Vol. 28 
The above inverse problem has been extensively studied [1]. The most powerful methods of investigation are the Gel'fand-Levitan [2] and Marchenko [3] ones. They both use the fact that (1.1) is an eigenvalue equation. However, the Marchenko method is much more direct than the Gel'fand-Levitan one which needs the introduction of a spectral function not easily connected with the input data.

More generally, we can try to describe the interactions through the equation

$$
y^{+\prime}+\left[E-V^{+}(E, x)\right] y^{+}=0, \quad x \geqq 0,
$$

in which the potential $V^{+}(E, x)$ depends on energy in some way. In particular, in this paper, we choose the following form for the energydependence:

$$
V^{+}(E, x)=U(x)+2 \sqrt{E} Q(x), \quad x \geqq 0 ;
$$

" $\sqrt{\mathrm{E}}$ " means the determination of the two-valued square root function, defined as (note the negative sign)

$$
\sqrt{E}=-|E|^{\frac{1}{2}} e^{\frac{i}{2} \operatorname{Arg} E}, \quad 0<\operatorname{Arg} E \leqq 2 \pi ;
$$

$U(x)$ and $Q(x)$ are complex valued functions. We note that, with the additional condition $U(x)=-Q^{2}(x),(1.2)$ reduces to the Klein-Gordon $s$-wave equation with the static potential $Q(x)$, for a particle of zero mass and of energy $\sqrt{\mathrm{E}}$.

It is useful to introduce the equation

$$
y^{-\prime \prime}+\left[E-V^{-}(E, x)\right] y^{-}=0, \quad x \geqq 0,
$$

with the potential

$$
V^{-}(E, x)=U(x)-2 \sqrt{E} Q(x), \quad x \geqq 0 ;
$$

i.e., we introduce the other determination " $-\sqrt{E}$ " of the two-valued square root function. Setting

$$
k=\sqrt{E},
$$

we write (1.2) and (1.5), (1.3) and (1.6), in the equivalent forms

$$
\begin{gathered}
y^{ \pm \prime}+\left[k^{2}-V^{ \pm}(k, x)\right] y^{ \pm}=0, \quad x \geqq 0,{ }^{1} \\
V^{ \pm}(k, x)=U(x) \pm 2 k Q(x), \quad x \geqq 0,
\end{gathered}
$$

where, because of our determination " $\sqrt{E}$ ", $\operatorname{Im} k<0$ or $k$ real positive. However, (1.8a) and (1.8b) have a meaning for every complex $k$; we sometimes use this fact in our work.

${ }^{1}$ The indices ${ }^{ \pm}$correspond to each other according to their position. This convention will be used throughout. 
In Section 2, assuming $U(x), Q(x)$ and $Q^{\prime}(x)$ continuous, and $x U(x)$, $Q(x)$ and $x Q^{\prime}(x)$ integrable for $x \geqq 0^{2}$, we examine the analytic properties, in the complex $k$-plane, of the regular solution $\varphi^{ \pm}(k, x)$ and of the Jost solution $f^{ \pm}(k, x)$ of (1.8); we also derive some useful bounds ${ }^{3}$. Assuming furthermore in Section 3 that the Jost function $f^{ \pm}(k)$ has only non real simple zeros in the half-plane $\operatorname{Im} k \leqq 0$ [4], we define as in the case $Q=0$ the binding energies $E_{n}^{ \pm}$and the scattering "matrix" $S^{ \pm}(k)(k>0)$; we note a "completion formula" 4 concerning the "admissible" solutions $\varphi^{+}(k, x)$ and $\varphi^{-}(k, x)$. The potentials $U(x)$ and $Q(x)$ have a physical meaning if the numbers $E_{n}^{+}$produced are real and negative, and if the function $S^{+}(k)(k>0)$ is absolutely bounded by 1 (this last condition is fulfilled if $\operatorname{Im} U(x) \leqq 0$ and $\operatorname{Im} Q(x) \leqq 0)$. However, as no additional mathematical difficulty arises, we also admit non-physical potentials in our study.

Our inverse problem is the construction of the potentials $U(x)$ and $Q(x)$, given $E_{n}^{+}$and $S^{+}(k)(k>0)$. That is, for given $E_{n}^{+}$and $S^{+}(k)(k>0)$, we discuss the existence, the uniqueness, and the explicit construction of $U$ and $Q$ in a certain class of functions. This problem has already been studied [5] by extending the Gel'fand-Levitan method. In this paper, we propose to solve it by a more direct approach generalizing the Marchenko method. The principal difference from the case $Q=0$ is that (1.2) is no longer an eigenvalue equation.

There is a simple plausibility argument for the existence of such a generalization. The problem which we want to study and the problem of a spinning particle in the scattering problem at fixed energy, as studied by Sabatier [6], are formally similar. Since it was possible in the latter case to generalize [6] the Gel'fand-Levitan-Regge-Newton procedure, we may hope, in our case, by using analogous extensions, to generalize the Marchenko procedure.

With the above assumptions on $U(x)$ and $Q(x)$, we show, in Section 4 , that $f^{ \pm}(k, x)$ is generated by two functions $F^{ \pm}(x)$ and $A^{ \pm}(x, t)$, and we derive, in Section 5, coupled integral equations connecting $F^{+}(x), F^{-}(x)$, $A^{+}(x, t)$ and $A^{-}(x, t)$ with two functions $z^{+}(x)(x>0)$ and $z^{-}(x)(x>0)$. These "fundamental functions" $z^{+}(x)$ and $z^{-}(x)$ are easily deduced from $E_{n}^{+}, E_{n}^{-}, S^{+}(k)(k>0), S^{-}(k)(k>0)$ and certain numbers $C_{n}^{+}$and $C_{n}^{-}$.

In Section 6, we investigate first the problem of solving the above integral equations given $z^{+}(x)$ and $z^{-}(x)$; we show how to construct

${ }^{2}$ These conditions can be weakened; in particular, the assumption " $U(x)$ continuous for $x=0$ " has been made for convenience only.

3 Throughout this paper, the term "bound" ("bounded") is often used instead of "absolute bound" ("absolutely bounded").

${ }^{4}$ We shall not use this "completion formula" in the following, though it is possible to derive formally the fundamental integral equations of our inverse problem from related formulas. 
potentials $U(x)$ and $Q(x)$ which yield given functions $z^{+}(x)$ and $z^{-}(x)$ as fundamental functions, and we give some specific examples. Finally, we apply these results to the solution of our inverse problem. In general, we may expect many solutions to the problem, since we may start from arbitrary values for the quantities $C_{n}^{+}, E_{n}^{-}, C_{n}^{-}$and $S^{-}(k)(k>0)$ to build the functions $z^{+}(x)$ and $z^{-}(x)$; but the degree of this indetermination has to be specified.

All the results we give can be refined, chiefly in the case of real potentials. These improvements, as well as applications of our method to other inverse problems in physics, will be the object of further studies. It would also be of interest to investigate the inverse problem for potentials with more complicated energy-dependence.

2. The Regular Solution $\varphi^{ \pm}(k, x)$ and the Jost Solution $f^{ \pm}(k, x)$

In this section, we establish some basic properties of particular solutions of the Schrödinger equation

where

$$
y^{ \pm \prime}+\left[k^{2}-V^{ \pm}(k, x)\right] y^{ \pm}=0, \quad x \geqq 0, \quad k=a+i b,
$$

$$
V^{ \pm}(k, x)=U(x) \pm 2 k Q(x) ;
$$

$U(x)$ and $Q(\mathrm{x})$ are in general complex valued functions and we assume:

Assumption I. $U(x)$ is continuous for $x \geqq 0$, and

$$
\int_{0}^{\infty} x|U(x)| d x<\infty .
$$

Assumption II. $Q(x)$ is continuously differentiable for $x \geqq 0$, and

$$
\begin{aligned}
& \int_{0}^{\infty}|Q(x)| d x<\infty, \\
& \int_{0}^{\infty} x\left|Q^{\prime}(x)\right| d x<\infty .
\end{aligned}
$$

As a consequence of these assumptions, $Q(\infty)=0$ and $x|Q(x)|$ is bounded for $x \geqq 0$.

The "regular solution" $\varphi^{ \pm}(k, x)$ is defined, as in the case $Q=0$, by the conditions

$$
\varphi^{ \pm}(k, 0)=0, \quad \varphi^{ \pm \prime}(k, 0)=1 .
$$

$\varphi^{ \pm}(k, x)$ is defined equivalently as the solution of the integral equation

$$
\varphi^{ \pm}(k, x)=\frac{\sin k x}{k}+\int_{0}^{x} \frac{\sin k(x-y)}{k} V^{ \pm}(k, y) \varphi^{ \pm}(k, y) d y
$$


in the class of functions continuous for $x \geqq 0$, and therefore, can be written as the sum of the Neumann series

where

$$
\varphi^{ \pm}(k, x)=\sum_{n=0}^{\infty} \varphi_{n}^{ \pm}(k, x),
$$

$$
\begin{gathered}
\varphi_{0}^{ \pm}(k, x)=\frac{\sin k x}{k}, \\
\varphi_{n}^{ \pm}(k, x)=\int_{0}^{x} \frac{\sin k(x-y)}{k} V^{ \pm}(k, y) \varphi_{n-1}^{ \pm}(k, y) d y, \quad n \geqq 1 .
\end{gathered}
$$

With the help of (2.6) we can prove:

Lemma 2.1. For each $x \geqq 0, \varphi^{ \pm}(k, x)$ is an entire function of $k$, and satisfies the following bounds ${ }^{5}$ :

where

$$
\left|\varphi^{ \pm}(k, x)\right| \leqq C e^{|b| x} \frac{x}{1+|k| x} e^{2 L(x)}, \quad x \geqq 0, \quad k \in \mathbb{C},
$$

$$
\begin{aligned}
& L(x)=\int_{0}^{x}[y|U(y)|+2|Q(y)|] d y, \\
& \left|\varphi^{ \pm}(k, x)-\left(H^{ \pm}(x) \frac{e^{i k x}}{2 i k}-H^{\mp}(x) \frac{e^{-i k x}}{2 i k}\right)\right| \leqq C \frac{e^{|b| x}}{|k|^{2}} e^{2 M(x)}, \\
& x \geqq 0, \quad|k| \geqq 1,
\end{aligned}
$$

where

$$
\begin{gathered}
H^{ \pm}(x)=e^{\mp i \int_{0}^{x} Q(y) d y}, \\
M(x)=\int_{0}^{x}\left(|U(y)|+2|Q(y)|+\left|Q^{\prime}(y)\right|\right) d y .
\end{gathered}
$$

We give the proof of (2.8) in Appendix A.1. The results (2.5), (2.6) and (2.7) are analogous to those obtained in the case $Q=0$ [7]. Note too that

$$
\varphi^{ \pm}(-k, x)=\varphi^{\mp}(k, x), \quad k \in \mathbb{C} .
$$

It is also possible to defined [8], for each complex $k$, a solution $J^{ \pm}(k, x)$ of (2.1) satisfying the asymptotic relations

$$
J^{ \pm}(k, x)=1+o(1), \quad J^{ \pm \prime}(k, x)=O(1), \quad \text { as } \quad x \rightarrow 0 .
$$

$\varphi^{ \pm}(k, x)$ and $J^{ \pm}(k, x)$ form a fundamental system of solutions of (2.1).

The "Jost solution" $f^{ \pm}(k, x)$ of $(2.1)$ is defined as usual by the asymptotic condition

$$
\lim _{x \rightarrow \infty} e^{i k x} f^{ \pm}(k, x)=1 \text {. }
$$

${ }^{5}$ In the following, we use $C$ as a general constant. It is not meant to have the same value everytime it is used. 
According to the Assumptions I and II, $f^{ \pm}(k, x)$ exists for $b \leqq 0 . f^{ \pm}(k, x)$ is defined equivalently as the solution of the integral equation

$$
f^{ \pm}(k, x)=e^{-i k x}+\int_{x}^{\infty} \frac{\sin k(y-x)}{k} V^{ \pm}(k, y) f^{ \pm}(k, y) d y
$$

in the class of functions continuous for $x \geqq 0$. It can therefore be expanded as follows:

where

$$
f^{ \pm}(k, x)=\sum_{n=0}^{\infty} f_{n}^{ \pm}(k, x),
$$

$$
f_{n}^{ \pm}(k, x)=\int_{x}^{\infty} \frac{\sin k(y-x)}{k} V^{ \pm}(k, y) f_{n-1}^{ \pm}(k, y) d y, \quad n \geqq 1 .
$$

With the help of these equations, we can prove:

Lemma 2.2. For each $x \geqq 0, f^{ \pm}(k, x)$, considered as a function of $k$, is continuous for $b \leqq 0$, analytic for $b<0$ and satisfies the following bounds:

where

$$
\left|f^{ \pm}(k, x)\right| \leqq e^{b x+2 N(x)}, \quad x \geqq 0, \quad b \leqq 0 ，
$$

$$
N(x)=\int_{x}^{\infty}(y|U(y)|+2|Q(y)|) d y,
$$

$$
\left|f^{ \pm}(k, x)-e^{-i k x} F^{ \pm}(x)\right| \leqq C \frac{e^{b x}}{|k|} e^{2 P(x)}, \quad x \geqq 0, \quad|k| \geqq 1,
$$

where

$$
\begin{gathered}
F^{ \pm}(x)=e^{\mp i \int_{r}^{\infty} Q(y) d y}, \\
P(x)=\int_{x}^{\infty}\left(|U(y)|+2|Q(y)|+\left|Q^{\prime}(y)\right|\right) d y .
\end{gathered}
$$

The proof of (2.15) is given in Appendix A.2. The proof of the other results quoted above presents no difficulty [9]. Note too that

$$
\lim _{x \rightarrow \infty} e^{i k x} f^{ \pm \prime}(k, x)=-i k .
$$

It is also possible to define [10], for $b \leqq 0$, a solution $g^{ \pm}(k, x)$ satisfying the condition

$$
\lim _{x \rightarrow \infty} e^{-i k x} g^{ \pm}(k, x)=1, \quad \lim _{x \rightarrow \infty} e^{-i k x} g^{ \pm^{\prime}}(k, x)=i k .
$$

$f^{ \pm}(k, x)$ and $g^{ \pm}(k, x)$ form a fundamental system of solutions of (2.1) for $k \neq 0$. We have the important relation

$$
g^{ \pm}(k, x)=f^{\mp}(-k, x), \quad b=0, \quad x \geqq 0 .
$$




\section{The Jost Function $f^{ \pm}(k)$, the Binding Energies $E_{n}^{ \pm}$ and the Scattering "Matrix" $S^{ \pm}(k)$}

In this section, by analogy with the case $Q=0$, we define, for $V^{ \pm}(k, x)$ $(b<0$ or $k>0)$, the binding energies $E_{n}^{ \pm}$and the scattering "matrix" $S^{ \pm}(k)(k>0)$.

$k$ being fixed, with $b<0$ or with $k>0$, we shall say that, for the potential $V^{ \pm}(k, x)$, the solution $\varphi^{ \pm}(k, x)$ represents a bound state if it is square integrable, a scattering state if it is bounded and not square integrable. We call these solutions the "admissible" solutions $\varphi^{ \pm}(k, x)$ of (2.1). To study them, it is useful to define ${ }^{6}$ the Jost function $f^{ \pm}(k)$ :

$$
f^{ \pm}(k)=W\left[f^{ \pm}(k, x), \varphi^{ \pm}(k, x)\right], \quad b \leqq 0,
$$

the function $g^{ \pm}(k)$ :

$$
g^{ \pm}(k)=W\left[g^{ \pm}(k, x), \varphi^{ \pm}(k, x)\right], \quad b \leqq 0,
$$

and the function $f_{J}^{ \pm}(k)$ :

$$
f_{J}^{ \pm}(k)=W\left[f^{ \pm}(k, x), J^{ \pm}(k, x)\right], \quad b \leqq 0 .
$$

We easily verify the formulas

$$
\begin{array}{ll}
W\left[\varphi^{ \pm}(k, x), J^{ \pm}(k, x)\right]=-1, & k \in \mathbb{C}, \\
W\left[f^{ \pm}(k, x), g^{ \pm}(k, x)\right]=2 i k, & b \leqq 0 .
\end{array}
$$

We deduce from the previous relations that $\varphi^{ \pm}(k, x)=\frac{1}{2 i k}\left[f^{ \pm}(k) g^{ \pm}(k, x)-g^{ \pm}(k) f^{ \pm}(k, x)\right], \quad b \leqq 0$ and $k \neq 0$,

and, with (2.18), that

$\varphi^{ \pm}(k, x)=\frac{1}{2 i k}\left[f^{ \pm}(k) f^{\mp}(-k, x)-f^{\mp}(-k) f^{ \pm}(k, x)\right], \quad k \in \mathbb{R}-\{0\}$.

In the same way, we find the formula

$$
f^{ \pm}(k, x)=f^{ \pm}(k) J^{ \pm}(k, x)-f_{J}^{ \pm}(k) \varphi^{ \pm}(k, x), \quad b \leqq 0,
$$

which yields, for $\mathrm{x}=0$, the useful equality

$$
f^{ \pm}(k)=f^{ \pm}(k, 0), \quad b \leqq 0 .
$$

Because of the Lemma 2.2, $f^{ \pm}(k)$ is continuous for $b \leqq 0$, analytic for $b<0$, and

$$
\lim _{|k| \rightarrow \infty} f^{ \pm}(k)=F^{ \pm}(0) .
$$

\footnotetext{
${ }^{6}$ We denote by $W\left(f_{1}, f_{2}\right)$ the wronskian of two functions $f_{1}$ and $f_{2}$.
} 
Using the asymptotic behaviour of $f^{ \pm}(k, x)$ and $g^{ \pm}(k, x)$ and the formula (3.6), we can assert that, relatively to the potential $V^{ \pm}(k, x)$ :

1. $\varphi^{ \pm}\left(k_{n}^{ \pm}, x\right)$ represents a bound state if and only if $k_{n}^{ \pm}$is a zero of $f^{ \pm}(k)$ for $b<0$; the numbers $E_{n}^{ \pm}=\left(k_{n}^{ \pm}\right)^{2}$ are the binding energies; for real $U(x)$ and $Q(x)$, a classic argument (similar to that used in the case $Q=0[7]$ to show that the binding energies are real and negative) leads to the following relation:

$$
\operatorname{Re} k_{n}^{ \pm}=\frac{\int_{0}^{\infty} Q(x)\left|\varphi^{ \pm}\left(k_{n}^{ \pm}, x\right)\right|^{2} d x}{\int_{0}^{\infty}\left|\varphi^{ \pm}\left(k_{n}^{ \pm}, x\right)\right|^{2} d x} ;
$$

2. $\varphi^{ \pm}(k, x)$ represents a scattering state if and only if $k$ is real and positive.

Now, we make an additional assumption:

Assumption III. $f^{ \pm}(k)$ has no real zero.

Let us remark that, for $U(x)$ and $Q(x)$ real, this condition is fulfilled if $f^{+}(k)$, and hence $f^{-}(k)$, does not vanish for $k=0$. We can then define the function

Clearly,

$$
S^{ \pm}(k)=\frac{f^{ \pm}(k)}{f^{\mp}(-k)}, \quad k \in \mathbb{R} .
$$

$$
\begin{gathered}
S^{ \pm}(0)=1, \\
\lim _{|k| \rightarrow \infty} S^{ \pm}(k)=\left[F^{ \pm}(0)\right]^{2} .
\end{gathered}
$$

Furthermore, as in the case of a complex energy-independent potential [11], we have the relation

$$
\left|S^{ \pm}(k)\right|^{2}=1+\frac{4 k}{\left|f^{\mp}(-k)\right|^{2}} \int_{0}^{\infty}\left|\varphi^{ \pm}(k, x)\right|^{2} \operatorname{Im} V^{ \pm}(k, x) d x .
$$

For $k>0, S^{ \pm}(k)$ represents the scattering "matrix" for $V^{ \pm}(k, x)$. The formula

$$
S^{ \pm}(k)=\frac{1}{S^{\mp}(-k)}, \quad k \in \mathbb{R},
$$

shows that $S^{+}(k)$ and $S^{-}(k)(k \in \mathbb{R})$ are determined given $S^{+}(k)$ and $S^{-}(k)(k>0)$. Note that the conditions $\operatorname{Im} U(x) \leqq 0$ and $\operatorname{Im} Q(x) \leqq 0$ imply, via (3.13c), that $\left|S^{+}(k)\right|(k>0)$ is bounded by 1 ; if also the binding energies $E_{n}^{+}$are real and negative, the potentials $U(x)$ and $Q(x)$ have a physical meaning. 
Using the properties of $f^{ \pm}(k)$ and the Assumption III, it is easy to prove that the number $N^{ \pm}$of zeros of $f^{ \pm}(k)(\operatorname{Im} k<0)$ is finite. In Section 5 we shall need the following additional assumption:

Assumption IV. The zeros of $f^{ \pm}(k)$ are simple.

We prove in Appendix $\mathrm{C}$ a "completion formula", concerning the "admissible" solutions $\varphi^{+}(k, x)$ and $\varphi^{-}(k, x)$, in the space of twice continuously differentiable functions $\psi(x)$ vanishing in the neighbourhood of $x=0$ and $x=\infty$.

To end, let us give conjugacy relations in the case where $U(x)$ and $Q(x)$ are real:

$$
\begin{aligned}
\overline{V^{ \pm}(k, x)} & =V^{ \pm}(\bar{k}, x), & & k \in \mathbb{C}, \\
\overline{\varphi^{ \pm}(k, x)} & =\varphi^{ \pm}(\bar{k}, x), & & k \in \mathbb{C}, \\
\overline{f^{ \pm}(k, x)} & =f^{\mp}(-\bar{k}, x), & & b \leqq 0, \\
\overline{f^{ \pm}(k)} & =f^{\mp}(-\bar{k}), & & b \leqq 0, \\
\overline{g^{ \pm}(k, x)} & =g^{\mp}(-\bar{k}, x), & & b \leqq 0, \\
\overline{g^{ \pm}(k)} & =g^{\mp}(-\bar{k}), & & b \leqq 0, \\
S^{ \pm}(k) \overline{S^{ \pm}(k)} & =1, & & k \in \mathbb{R} .
\end{aligned}
$$

As a consequence of (3.18), if $k_{n}^{ \pm}$is a zero of $f^{ \pm}(k),-\overline{k_{n}^{ \pm}}$is a zero of $f^{\mp}(k)$; so, $N^{+}$and $N^{-}$are equal and we can number the zeros of $f^{+}(k)$ and $f^{-}(k)$ in such a way that

$$
k_{n}^{\mp}=-\overline{k_{n}^{ \pm}} .
$$

We have too

$$
\left.\overline{\frac{d}{d k} f^{+}(k)}\right|_{k=k_{n}^{+}}=-\left.\frac{d}{d k} f^{-}(k)\right|_{k=k_{n}^{-}} .
$$

\section{A Useful Expression for $f^{ \pm}(k, x)$}

With the Assumptions I and II, we show that the Jost solution $f^{ \pm}(k, x)$ can be conveniently generated by two auxiliary functions $F^{ \pm}(x)$ and $A^{ \pm}(x, t)$ and we study closely related questions. The principal results of the section are the Theorems 4.1 and 4.2. They will be very useful later.

We obtain a preliminary result very simply. From the bounds (2.14) and (2.15) it follows that

$$
\int_{-\infty}^{\infty}\left|f^{ \pm}(a+i b, x)-F^{ \pm}(x) e^{-i a x} e^{b x}\right|^{2} d a=O\left(e^{2 b x}\right), \quad b \leqq 0, \quad x \geqq 0 .
$$


We deduce from the Plancherel theorem that there exists a function $\tilde{A}^{ \pm}(x, t)$, belonging to the class $L^{2}(-\infty, \infty)$ in $t$, such that

$$
f^{ \pm}(k, x)-F^{ \pm}(x) e^{-i k x}=\lim _{n \rightarrow \infty} . \int_{-n}^{+n} \tilde{A}^{ \pm}(x, t) e^{-i k t} d t, \quad \text { for } \quad b=0, \quad x \geqq 0 .
$$

Furthermore, according to a Titchmarsh theorem [12], we conclude from the formula (4.1) and the Lemma 2.2 that

$$
\tilde{A}^{ \pm}(x, t)=0, \text { for } t<x .
$$

As a consequence, $f^{ \pm}(k, x)$ may be written in the form

$$
f^{ \pm}(k, x)=F^{ \pm}(x) e^{-i k x}+\underset{n \rightarrow \infty}{\lim .} \int_{x}^{n} \tilde{A}^{ \pm}(x, t) e^{-i k t} d t, \quad \text { for } \quad b=0, x \geqq 0 .
$$

The relation (4.4), though formally simple, is not easy to use, and gives little information about the function $\tilde{A}^{ \pm}(x, t)$.

We now obtain a more accurate result by another method. In the following, we denote by $\sigma^{7}$ any non-increasing positive function, defined for $x \geqq 0$, such that

$$
\int_{0}^{\infty} \sigma(x) d x<\infty
$$

Let us introduce the class $\mathfrak{A}$ of functions $A^{ \pm}(x, t)$ defined and continuous with respect to $(x, t)$ for $t \geqq x \geqq 0$, and satisfying the condition

$$
\left|A^{ \pm}(x, t)\right| \leqq \sigma\left(\frac{x+t}{2}\right)
$$

where $\sigma$ may depend on $A^{ \pm}$; as a consequence, $A^{ \pm}(x, t)$ belongs to the classes $L^{1}(x, \infty)$ and $L^{2}(x, \infty)$ in $t$.

First, we prove the following lemma:

Lemma 4.1. Suppose that $U(x)$ and $Q(x)$ satisfy the Assumptions $I$ and II, that $A^{ \pm}(x, t)$ is a function belonging to $\mathfrak{A}$ and that $\tilde{F}^{ \pm}(x)$ is a continuous and bounded function. Define $\tilde{f}^{ \pm}(k, x)$ as

$$
\tilde{f}^{ \pm}(k, x)=\tilde{F}^{ \pm}(x) e^{-i k x}+\int_{x}^{\infty} A^{ \pm}(x, t) e^{-i k t} d t, \quad x \geqq 0, \quad b \leqq 0 .
$$

\footnotetext{
7 The symbol $\sigma$ is not meant to designate the same function everytime it is used.
} 
Then $\tilde{f}^{ \pm}(k, x)$ is the Jost solution $f^{ \pm}(k, x)$ of the Schrödinger equation (2.1) if and only if

a) $\tilde{F}^{ \pm}(x) \equiv F^{ \pm}(x), \quad x \geqq 0$,

b) $A^{ \pm}(x, t)$ is a solution, in the class $\mathfrak{A}$, of the integral equation

$$
\begin{aligned}
& A^{ \pm}(x, t)=\frac{1}{2} \int_{\frac{x+t}{2}}^{\infty} F^{ \pm}(y) U(y) d y+\frac{1}{2} \int_{x}^{\frac{x+t}{2}} U(y) d y \int_{t+x-y}^{t+y-x} A^{ \pm}(y, u) d u \\
&+\frac{1}{2} \int_{\frac{x+t}{2}}^{\infty} U(y) d y \int_{y}^{t+y-x} A^{ \pm}(y, u) d u \pm \frac{i}{2} F^{ \pm}\left(\frac{x+t}{2}\right) Q\left(\frac{x+t}{2}\right) \\
& \mp i \int_{x}^{\infty} Q(y) A^{ \pm}(y, t+y-x) d y \pm i \int_{x}^{\frac{x+t}{2}} Q(y) A^{ \pm}(y, t+x-y) d y, \\
& t \geqq x \geqq 0 .
\end{aligned}
$$

To prove the necessity of the conditions, we replace $f^{ \pm}(k, x)$ by $\tilde{f}^{ \pm}(k, x)$ in (2.12) and make use of the formulas

$$
\begin{aligned}
& \frac{\sin k(y-x)}{k} e^{-i k y}=\frac{1}{2} \int_{x}^{2 y-x} e^{-i k t} d t, \\
& \frac{\sin k(y-x)}{k} e^{-i k u}=\frac{1}{2} \int_{u+x-y}^{u+y-x} e^{-i k t} d t
\end{aligned}
$$

we obtain

$$
\begin{aligned}
\int_{x}^{\infty} A^{ \pm}(x, y) e^{-i k y} d y= & \left(1-\tilde{F}^{ \pm}(x)\right) e^{-i k x}+\frac{1}{2} \int_{x}^{\infty} V^{ \pm}(k, y) \tilde{F}^{ \pm}(y) d y \int_{x}^{2 y-x} e^{-i k t} d t \\
& +\frac{1}{2} \int_{x}^{\infty} V^{ \pm}(k, y) d y \int_{y}^{\infty} A^{ \pm}(y, u) d u \int_{u+x-y}^{u+y-x} e^{-i k t} d t
\end{aligned}
$$

Clearly, the hypotheses of the Lemma 4.1 allow the interchange of the orders of integration in the integrals on the right hand side of (4.10). 
This yields the following equality:

$$
\begin{aligned}
& \int_{x}^{\infty} A^{ \pm}(x, y) e^{-i k y} d y=\left(1-\tilde{F}^{ \pm}(x)\right) e^{-i k x}+\int_{x}^{\infty} e^{-i k t} d t\left[\frac{1}{2} \int_{\frac{x+t}{2}}^{\infty} \tilde{F}^{ \pm}(y) U(y) d y\right. \\
& \left.+\frac{1}{2} \int_{x}^{\frac{x+t}{2}} U(y) d y \int_{t+x-y}^{t+y-x} A^{ \pm}(y, u) d u+\frac{1}{2} \int_{\frac{x+t}{2}}^{\infty} U(y) d y \int_{y}^{t+y-x} A^{ \pm}(y, u) d u\right] \\
& \pm \int_{x}^{\infty} k e^{-i k t} d t\left[\int_{\frac{x+t}{2}}^{\infty} \tilde{F}^{ \pm}(y) Q(y) d y\right. \\
& \left.\quad \frac{\int_{x}^{2+t}}{2} Q(y) d y \int_{t+x-y}^{t+y-x} A^{ \pm}(y, u) d u+\int_{\frac{x+t}{2}}^{\infty} Q(y) d y \int_{y}^{t+y-x} A^{ \pm}(y, u) d u\right] .
\end{aligned}
$$

We integrate by parts the last term of $(4.11) ;(4.11)$ then takes the form

$$
\begin{aligned}
{\left[\tilde{F}^{ \pm}(x)\right.} & \left.-1 \pm i \int_{x}^{\infty} \tilde{F}^{ \pm}(y) Q(y) d y\right] e^{-i k x} \\
& +\int_{x}^{\infty} e^{-i k t} d t\left[A^{ \pm}(x, t)-\frac{1}{2} \int_{\frac{x+t}{2}}^{\infty} \tilde{F}^{ \pm}(y) U(y) d y\right. \\
& -\frac{1}{2} \int_{x}^{\frac{x+t}{2}} U(y) d y \int_{t+x-y}^{t+y-x} A^{ \pm}(y, u) d u \\
& -\frac{1}{2} \int_{\frac{x+t}{2}}^{\infty} U(y) d y \int_{y}^{t+y-x} A^{ \pm}(y, u) d u \mp \frac{i}{2} \tilde{F}^{ \pm}\left(\frac{x+t}{2}\right) Q\left(\frac{x+t}{2}\right) \\
& \left. \pm i \int_{x}^{\infty} Q(y) A^{ \pm}(y, t+y-x) d y \mp i \int_{x}^{\frac{x+t}{2}} Q(y) A^{ \pm}(y, t+x-y) d y\right]=0 .
\end{aligned}
$$

The first member of (4.12) appearing as a Fourier transform, the conditions (4.8) follow easily. It is trivial to show that (4.8) is sufficient for $f^{ \pm}(k, x)$ to be the Jost solution of (2.1).

Let us now study the integral Eq. (4.8b). For this, we apply the method of successive approximations. We define

$$
A_{0}^{ \pm}(x, t)=\frac{1}{2} \int_{\frac{x+t}{2}}^{\infty} F^{ \pm}(y) U(y) d y \pm \frac{i}{2} F^{ \pm}\left(\frac{x+t}{2}\right) Q\left(\frac{x+t}{2}\right),
$$


and, for $n \geqq 1$,

$$
\begin{aligned}
A_{n}^{ \pm}(x, t) & =\frac{1}{2} \int_{x}^{\frac{x+t}{2}} U(y) d y \int_{t+x-y}^{t+y-x} A_{n-1}^{ \pm}(y, u) d u \\
& +\frac{1}{2} \int_{\frac{x+t}{2}}^{\infty} U(y) d y \int_{y}^{t+y-x} A_{n-1}^{ \pm}(y, u) d u \\
& \mp i \int_{x}^{\infty} Q(y) A_{n-1}^{ \pm}(y, t+y-x) d y \pm i \int_{x}^{\frac{x+t}{2}} Q(y) A_{n-1}^{ \pm}(y, t+x-y) d y .
\end{aligned}
$$

It is possible to derive by induction a bound for $A_{n}^{ \pm}$:

where

$$
\left|A_{n}^{ \pm}(x, t)\right| \leqq \frac{m}{2} \sigma_{v}\left(\frac{x+t}{2}\right) \frac{[N(x)]^{n}}{n !}, \text { for } n \in N,
$$

$$
\begin{gathered}
m=e^{\int_{0}^{\infty}|\operatorname{Im} Q(y)| d y}, \\
\sigma_{v}(x)=\int_{x}^{\infty}\left[|U(y)|+\left|Q^{\prime}(y)\right|\right] d y,
\end{gathered}
$$

$N(x)$ is defined by $(2.14 \mathrm{~b})$.

As a consequence, the series

$$
A^{ \pm}(x, t)=\sum_{n=0}^{\infty} A_{n}^{ \pm}(x, t)
$$

converges uniformly for $t \geqq x \geqq 0$. On the other hand, we see (by induction) that $A_{n}^{ \pm}(x, t)$ is continuous for $t \geqq x \geqq 0$; it follows that $A^{ \pm}(x, t)$ is continuous for $t \geqq x \geqq 0$. From (4.14a), we derive a bound for $A^{ \pm}(x, t)$ :

$$
\left|A^{ \pm}(x, t)\right| \leqq \frac{m}{2} \sigma_{v}\left(\frac{x+t}{2}\right) e^{N(x)} .
$$

Since

$$
\int_{0}^{\infty} \sigma_{v}(x) d x \leqq \int_{0}^{\infty} y\left[|U(y)|+\left|Q^{\prime}(y)\right|\right] d y<\infty,
$$

we can finally conclude that $A^{ \pm}(x, t)$ belongs to the class $\mathfrak{A}$. Lastly, inserting the series $A^{ \pm}(x, t)$, defined by (4.15), in the integral equation (4.8 $\mathrm{b})$, we verify that $A^{ \pm}(x, t)$ is a solution of this equation in the class $\mathfrak{A}$. If $A^{\prime \pm}(x, t)$ is another solution of $(4.8 \mathrm{~b})$ in the class $\mathfrak{A}$, one can prove by iteration the inequality

$$
\left|A^{\prime \pm}(x, t)-A^{ \pm}(x, t)\right| \leqq \sigma\left(\frac{x+t}{2}\right) \frac{[N(x)]^{n}}{n !}, \text { for } n \in N
$$


Hence:

$$
A^{\prime \pm}(x, t) \equiv A^{ \pm}(x, t) .
$$

We can now state the following result:

Lemma 4.2. With the Assumptions $I$ and II, the integral equation (4.8b) has a unique solution in the class $\mathfrak{A}$.

Lemmas (4.1) and (4.2) yield the following theorem:

Theorem 4.1. With the Assumptions I and II, there exists a unique function $A^{ \pm}$belonging to the class $\mathfrak{U}$ and a unique continuous and bounded function $\tilde{F}^{ \pm}$such that the Jost solution $f^{ \pm}(k, x)$ may be expanded in the form

$$
f^{ \pm}(k, x)=\tilde{F}^{ \pm}(x) e^{-i k x}+\int_{x}^{\infty} A^{ \pm}(x, t) e^{-i k t} d t, \quad b \leqq 0, x \geqq 0 ;
$$

$\tilde{F}^{ \pm}(x) \equiv F^{ \pm}(x)$ and $A^{ \pm}(x, t)$ is the unique solution in the class $\mathfrak{A}$ of $(4.8 \mathrm{~b})$.

The formulation (4.20) generalizes that given by Marchenko [13]. It is easy to verify using (4.13) that, for $U(x)$ and $Q(x)$ real, we have

$$
\overline{A^{+}(x, t)}=A^{-}(x, t) \text {. }
$$

Note too the identity [14]

$$
A^{ \pm}(x, t)=\tilde{A}^{ \pm}(x, t), \text { almost everywhere in } t .
$$

Let us further investigate $A^{ \pm}$. Starting from the expansion (4.15), we show in the Appendix B that the first partial derivatives exist and are continuous for $t \geqq x \geqq 0$, and obey

where

$$
\left.\begin{array}{l}
\left|\frac{\partial}{\partial x} A^{ \pm}(x, t)\right| \leqq C\left[\sigma_{v}^{2}(x)+W\left(\frac{x+t}{2}\right)\right], \\
\left|\frac{\partial}{\partial t} A^{ \pm}(x, t)\right| \leqq C\left[\sigma_{v}^{2}(x)+W\left(\frac{x+t}{2}\right)\right],
\end{array}\right\}
$$

For convenience, we now introduce the variables

$$
\xi=\frac{x+t}{2}, \quad \eta=\frac{x-t}{2}
$$

and the function

$$
\alpha^{ \pm}(\xi, \eta)=A^{ \pm}(x, t)
$$


With these variables, the Eq. $(4.8 \mathrm{~b})$ can be written as

$$
\begin{gathered}
\alpha^{ \pm}(\xi, \eta)=\frac{1}{2} \int_{\xi}^{\infty} F^{ \pm}(y) U(y) d y-\int_{\xi}^{\infty} d v \int_{0}^{\eta} U(v+s) \alpha^{ \pm}(v, s) d s \pm \frac{i}{2} F^{ \pm}(\xi) Q(\xi) \\
\mp i \int_{\xi}^{\infty} Q(u+\eta) \alpha^{ \pm}(u, \eta) d u \mp i \int_{0}^{\eta} Q(u+\xi) \alpha^{ \pm}(\xi, u) d u \\
\xi \geqq 0, \quad \eta \leqq 0 \text { and } \xi+\eta \geqq 0
\end{gathered}
$$

Clearly, $\frac{\partial}{\partial \xi} \alpha^{ \pm}(\xi, \eta), \frac{\partial}{\partial \eta} \alpha^{ \pm}(\xi, \eta)$ and the mixed derivative $\frac{\partial^{2} \alpha^{ \pm}}{\partial \xi \partial \eta}(\xi, \eta)$ exist, and

$\frac{\partial^{2} \alpha^{ \pm}}{\partial \xi \partial \eta}(\xi, \eta)-U(\xi+\eta) \alpha^{ \pm}(\xi, \eta) \mp i\left(\frac{\partial}{\partial \eta}-\frac{\partial}{\partial \xi}\right)\left[Q(\xi+\eta) \alpha^{ \pm}(\xi, \eta)\right]=0$.

Setting $t=x$ and $\eta=0$ in (4.8b) and (4.25) respectively, and differentiating both sides of the results, we find respectively

$$
\begin{aligned}
& F^{ \pm \prime}(x)-2 \frac{d}{d x} A^{ \pm}(x, x) \pm 2 i Q(x) A^{ \pm}(x, x)-F^{ \pm}(x) U(x)=0, \\
& F^{ \pm \prime}(\xi)-2 \frac{d}{d \xi} \alpha^{ \pm}(\xi, 0) \pm 2 i Q(\xi) \alpha^{ \pm}(\xi, 0)-F^{ \pm}(\xi) U(\xi)=0 .
\end{aligned}
$$

Conversely, suppose that $A^{ \pm}(x, t)$ belongs to the class $\mathfrak{A}$, that the derivatives $\frac{\partial}{\partial \xi} \alpha^{ \pm}(\xi, \eta), \frac{\partial}{\partial \eta} \alpha^{ \pm}(\xi, \eta), \frac{\partial^{2} \alpha^{ \pm}}{\partial \xi \partial \eta}(\xi, \eta)$ exist and are continuous with respect to $(\xi, \eta)$, and that the Eqs. (4.26) and (4.27b) be satisfied. Then, we can easily show that $\alpha^{ \pm}(\xi, \eta)$ is a solution of the integral equation (4.25), and $A^{ \pm}(x, t)$ is a solution of the integral equation (4.8b). Furthermore, if $A^{ \pm}(x, t)$ has second partial continuous derivatives, we notice that

$$
\frac{\partial^{2} \alpha^{ \pm}}{\partial \xi \partial \eta}(\xi, \eta)=\frac{\partial^{2} A^{ \pm}}{\partial x^{2}}(x, t)-\frac{\partial^{2} A^{ \pm}}{\partial t^{2}}(x, t),
$$

and that the Eq. (4.26) takes the form:

$\frac{\partial^{2} A^{ \pm}}{\partial x^{2}}(x, t)-\frac{\partial^{2} A^{ \pm}}{\partial t^{2}}(x, t)-U(x) A^{ \pm}(x, t) \pm 2 i Q(x) \frac{\partial}{\partial t} A^{ \pm}(x, t)=0$.

Using the Lemma 4.1, we can prove the following theorem which will be useful in Section 6: 
Theorem 4.2. Suppose that $U(x)$ and $Q(x)$ satisfy Assumptions I and II, and that the function $\tilde{f}^{ \pm}(k, x)$ defined in (4.7) is such that

a) $\tilde{F}^{ \pm}(x) \equiv F^{ \pm}(x)$

b) $A^{ \pm}(x, t)$ belongs to $\mathfrak{A}$,

c) the derivatives $\frac{\partial A^{ \pm}}{\partial x}(x, t), \frac{\partial A^{ \pm}}{\partial t}(x, t), \frac{\partial^{2} A^{ \pm}}{\partial x^{2}}(x, t)$ and $\frac{\partial^{2} A^{ \pm}}{\partial t^{2}}(x, t)$ exist and are continuous with respect to $(x, t)$,

d) the Eqs. (4.29) and (4.27a) be satisfied,

e) $\lim _{N \rightarrow \infty}\left(\sup _{t+x \geqq N}\left|\frac{\partial A^{ \pm}}{\partial x}(x, t)\right|\right)=0$,

$$
\lim _{N \rightarrow \infty}\left(\sup _{t+x \geqq N}\left|\frac{\partial A^{ \pm}}{\partial t}(x, t)\right|\right)=0 \text {; }
$$

then $\tilde{f}^{ \pm}(k, x)$ is the Jost solution of the Schrödinger equation (2.1).

\section{Derivation of Fundamental Integral Equations}

In this section, we make the Assumptions I, II, III and IV. We derive coupled integral relations connecting $\mathrm{F}^{+}(x), \mathrm{F}^{-}(x), A^{+}(x, t)$ and $A^{-}(x, t)$ with two functions $z^{+}(x)$ and $z^{-}(x)$ directly determined by $E_{n}^{+}, E_{n}^{-}$, $S^{+}(k)(k>0), S^{-}(k)(k>0)$ and certain numbers $C_{n}^{+}$and $C_{n}^{-}$(see 5.12). This result is precisely stated in the Theorem 5.1.

Writing the formula (4.20) for the case $x=0$, we find

$$
\begin{aligned}
f^{ \pm}(k) & =F^{ \pm}(0)+\int_{0}^{\infty} A^{ \pm}(0, t) e^{-i k t} d t, \quad k \in \mathbb{R}, \\
f^{\mp}(-k) & =F^{\mp}(0)+\int_{-\infty}^{0} A^{\mp}(0,-t) e^{-i k t} d t, \quad k \in \mathbb{R},
\end{aligned}
$$

where $A^{ \pm}(0, t)$ belongs to $L^{1}(0, \infty)$ and $A^{\mp}(0,-t)$ belongs to $L^{1}(-\infty, 0)$. Theorems on Fourier transforms of integrable functions, and particularly a Wiener-Levy theorem [15], show that there exists a function $s^{ \pm}(y)$ defined and integrable on $\mathbb{R}$ such that

$$
S^{ \pm}(k)=\left[F^{ \pm}(0)\right]^{2}+\int_{-\infty}^{\infty} S^{ \pm}(t) e^{-i k t} d t
$$

Let us now consider the equality derived from (3.7) by dividing both sides by $\frac{f^{ \pm}(k)}{2 i k}$ :

$$
2 i k \frac{\varphi^{ \pm}(k, x)}{f^{ \pm}(k)}=f^{\mp}(-k, x)-S^{\mp}(-k) f^{ \pm}(k, x), \quad k \in \mathbb{R} .
$$


Substituting (4.20) and (5.3) in (5.4), we obtain

$$
\begin{aligned}
& 2 k \varphi^{ \pm}(k, x)\left[\frac{1}{f^{ \pm}(k)}-F^{\mp}(0)\right] \\
& +2 i k F^{\mp}(0)\left[\varphi^{ \pm}(k, x)-\left(H^{ \pm}(x) \frac{e^{i k x}}{2 i k}-H^{\mp}(x) \frac{e^{-i k x}}{2 i k}\right)\right] \\
& \quad=-F^{ \pm}(x) e^{-i k x} \int_{-\infty}^{\infty} s^{\mp}(t) e^{i k t} d t-\left[F^{\mp}(0)\right]^{2} \int_{x}^{\infty} A^{ \pm}(x, t) e^{-i k t} d t \\
& \quad+\int_{x}^{\infty} A^{\mp}(x, t) e^{i k t} d t-\left(\int_{-\infty}^{\infty} s^{\mp}(t) e^{i k t} d t\right)\left(\int_{x}^{\infty} A^{ \pm}(x, t) e^{-i k t} d t\right) .
\end{aligned}
$$

The right hand side of (5.5) is easily written in the form of the Fourier transform of a function $B^{ \pm}(x, t)$ integrable with respect to $t$ :

$$
B^{\mp}(x, t)=\left\{\begin{array}{cc}
-\left[F^{\mp}(0)\right]^{2} A^{ \pm}(x,-t)-F^{ \pm}(x) s^{\mp}(t+x) & \\
-\int_{x}^{\infty} s^{\mp}(t+u) A^{ \pm}(x, u) d u, & t \leqq-x, \\
-F^{ \pm}(x) s^{\mp}(t+x) & \\
-\int_{x}^{\infty} s^{\mp}(t+u) A^{ \pm}(x, u) d u, & -x<t<x, \\
A^{\mp}(x, t)-F^{ \pm}(x) s^{\mp}(t+x) & \\
-\int_{x}^{\infty} s^{\mp}(t+u) A^{ \pm}(x, u) d u, & t \geqq x .
\end{array}\right.
$$

If the Fourier transform $\tilde{B}^{\mp}(x, t)$ of the left hand side of $(5.5)$ exists as a Cauchy principal-value in some interval of $t$, then $\tilde{B}^{\mp}(x, t)$ and $B^{\mp}(x, t)$ must coincide almost everywhere in this interval. We therefore evaluate the quantity

$$
\begin{aligned}
\tilde{B}_{R}^{\mp}(x, t) & =\frac{1}{2 \pi} \int_{-R}^{+R}\left[2 i k \varphi^{ \pm}(k, x)\left(\frac{1}{f^{ \pm}(k)}-F^{\mp}(0)\right)\right. \\
& \left.+2 i k F^{\mp}(0)\left(\varphi^{ \pm}(k, x)-\left(H^{ \pm}(x) \frac{e^{i k x}}{2 i k}-H^{\mp}(x) \frac{e^{-i k x}}{2 i k}\right)\right)\right] e^{-i k t} d k
\end{aligned}
$$

Let us consider the same integral computed along a closed path consisting of the real segment $[-R,+R]$ and the half circle $|k|=R$ contained in the lower half of the complex $k$-plane. Since $\varphi^{ \pm}(k, u)$ and $f^{ \pm}(k)$ are continuous for $\operatorname{Im} k \leqq 0$ and analytic for $\operatorname{Im} k<0$, and since the Assumption III holds, we can apply the theorem of residues. Furthermore, 
thanks to the bounds (2.7a), (2.8a) and the relation (3.10), we see, using one of Jordan's lemmas, that, in the case $t>x \geqq 0$, the integral along the half circle vanishes as $R \rightarrow \infty$. We conclude that the quantity

$$
\tilde{B}^{\mp}(x, t)=\lim _{R \rightarrow \infty} \tilde{B}_{R}^{\mp}(x, t)
$$

exists for $t>x \geqq 0$ and is given by

$$
\tilde{B}^{\mp}(x, t)=i \sum_{n=1}^{N^{ \pm}} \operatorname{Res}_{k=k_{n}^{ \pm}}\left[2 i k \frac{\varphi^{ \pm}(k, x)}{f^{ \pm}(k)} e^{-i k t}\right], \quad t>x \geqq 0 .
$$

Using the Assumption IV, we obtain

$$
\tilde{B}^{\mp}(x, t)=i \sum_{n=1}^{N^{ \pm}} \frac{2 i k_{n}^{ \pm} \varphi^{ \pm}\left(k_{n}^{ \pm}, x\right) e^{-i k_{n}^{ \pm} t}}{\left.\frac{d}{d k} f^{ \pm}(k)\right|_{k=k_{n}^{ \pm}}}, \quad t>x \geqq 0 .
$$

With the help of the relations (3.6) and (4.20), we write

where

$$
\tilde{B}^{\mp}(x, t)=\sum_{n=1}^{N^{ \pm}} C_{n}^{\mp} f^{ \pm}\left(k_{n}^{ \pm}, x\right) e^{-i k_{n}^{ \pm} t}, \quad t>x \geqq 0,
$$

$$
C_{n}^{\mp}=\frac{-i g^{ \pm}\left(k_{n}^{ \pm}\right)}{\left.\frac{d}{d k} f^{ \pm}(k)\right|_{k=k_{n}^{ \pm}}},
$$

and

$\tilde{B}^{\mp}(x, t)=F^{ \pm}(x) p^{\mp}(x+t)+\int_{x}^{\infty} A^{ \pm}(x, u) p^{\mp}(u+t) d u, \quad t>x \geqq 0$,

where

$$
p^{\mp}(x)=\sum_{n=1}^{N^{\mp}} C_{n}^{\mp} e^{-i k_{n}^{ \pm} x} .
$$

So, we have shown

$$
\tilde{B}^{\mp}(x, t)=B^{\mp}(x, t),
$$

for almost every $t(t>x \geqq 0)$. It is always possible to choose the function $s^{ \pm}(t)$ of (5.3) in such way that the following equality:

$$
\begin{aligned}
s^{\mp}(t)=F^{\mp}(0) A^{\mp}(0, t) & -p^{\mp}(t)-F^{\mp}(0) \int_{0}^{\infty} A^{ \pm}(0, u) s^{\mp}(u+t) d u \\
& -F^{\mp}(0) \int_{0}^{\infty} A^{ \pm}(0, u) p^{\mp}(u+t) d u,
\end{aligned}
$$

deduced from (5.15), (5.13) and (5.6) by putting $x=0$, holds everywhere for $t \geqq 0$. Since $s^{ \pm}(t)$ and $p^{ \pm}(t)$ are integrable for $t \geqq 0, p^{ \pm}(t)$ is continuous for $t \geqq 0$, and $A^{ \pm}(0, t)$ is continuous and bounded for $t \geqq 0$, it is easy to 
see that $s^{ \pm}(t)$ is continuous for $t \geqq 0$. As a consequence, $B^{ \pm}(x, t)$ is continuous with respect to $(x, t)$ for $t \geqq x \geqq 0$. A glance at (5.13), shows that the same is true for $\tilde{B}^{ \pm}(x, t)$. Therefore, the equality (5.15) holds for $t \geqq x \geqq 0$. We finally obtain two coupled integral equations:

where

$$
A^{ \pm}(x, t)=F^{\mp}(x) z^{ \pm}(x+t)+\int_{x}^{\infty} A^{\mp}(x, u) z^{ \pm}(u+t) d u, \quad t \geqq x \geqq 0,
$$

$$
z^{ \pm}(x)=s^{ \pm}(x)+p^{ \pm}(x) .
$$

$z^{+}$and $z^{-}$will be called the "fundamental functions" associated to $U$ and $Q$. We see from the formula

$$
s^{ \pm}(x)=\lim _{R \rightarrow \infty} \frac{1}{2 \pi} \int_{-R}^{+R}\left[S^{ \pm}(k)-F^{ \pm}(0)^{2}\right] e^{i k x} d k, \quad x>0,
$$

which will be proved later on, and from the formulas (3.13), (3.14) and (5.14) that the fundamental functions are determined by the data of $E_{n}^{+}$, $E_{n}^{-}, S^{+}(k)(k>0), S^{-}(k)(k>0)$ and the numbers $C_{n}^{+}$and $C_{n}^{-}$.

On the other hand, we easily derive from (4.20) the relation

$$
F^{+}(x)-F^{-}(x)=\int_{x}^{\infty}\left[A^{-}(x, t)-A^{+}(x, t)\right] d t,
$$

and from $(2.15 \mathrm{~b})$ the relations

$$
\begin{gathered}
F^{+}(x) F^{-}(x)=1, \\
\lim _{x \rightarrow \infty} F^{ \pm}(x)=1 .
\end{gathered}
$$

The Eqs. (5.17), (5.20) and (5.21) replace the fundamental equation obtained by Marchenko [16] and are the analogues of equations given by Sabatier [17] for the scattering of a spinning particle. In the next section, they will be the starting point of the investigation of our inverse problem.

In what follows, we consider $z^{ \pm}(x)$. It is clear first that $z^{ \pm}(x)$ is continuous for $x \geqq 0$ and integrable on $[0, \infty$ [. In order to obtain a bound for $z^{ \pm}$, we shall need the following classic result $(R)$ [18]:

$(R)$ : "Let $f(x)$ and $g(x)$ be non-negative functions in the interval $a \leqq x \leqq b \leqq \infty$, and suppose that $g(x)$ and $f(x) g(x)$ are integrable in this interval. If

$$
f(x) \leqq c+\int_{x}^{b} f(t) g(t) d t, \quad a \leqq x \leqq b,
$$

where $c$ is a positive constant, then

$$
f(x) \leqq c e^{\int_{x}^{b} g(t) d t}, a \leqq x \leqq b . "
$$


Using the bound (4.16) in the Eq. (5.17), we obtain

$$
\left|z^{ \pm}(x+t)\right| \leqq c \sigma_{v}\left(\frac{x+t}{2}\right)+c \int_{x}^{\infty}\left|z^{ \pm}(u+t)\right| \sigma_{v}\left(\frac{x+u}{2}\right) d u .
$$

Suppose first that $\sigma_{v}(u)$ does not vanish for any positive value of $u$; then,

$$
\begin{aligned}
& \left|z^{ \pm}(x+t) \sigma_{v}^{-1}\left(\frac{x+t}{2}\right)\right| \leqq c \\
& \quad+c \sigma_{v}^{-1}(t) \int_{x}^{\infty}\left[z^{ \pm}(u+t) \sigma_{v}^{-1}\left(\frac{u+t}{2}\right)\right]\left[\sigma_{v}\left(\frac{u+t}{2}\right) \sigma_{v}\left(\frac{u}{2}\right)\right] d u .
\end{aligned}
$$

Using $(R)$ we have:

$$
\left|z^{ \pm}(x+t) \sigma_{v}^{-1}\left(\frac{x+t}{2}\right)\right| \leqq c e^{c \sigma_{v}^{-1}(t) \int_{x}^{\infty} \sigma_{v}\left(\frac{u+t}{2}\right)} \sigma_{\sigma_{v}}\left(\frac{u}{2}\right) d u .
$$

Setting $t=x$ in (5.23), it is easy to obtain the bound

$$
\left|z^{ \pm}(x)\right| \leqq c \sigma_{v}\left(\frac{x}{2}\right), \quad x \geqq 0 .
$$

If now $\sigma_{v}(u)$ vanishes for some positive value of $u$, there exists some $u_{0}>0$ such that $\sigma_{v}(u)=0$ for $u \geqq u_{0}$ and $\sigma_{v}(u) \neq 0$ for $0 \leqq u<u_{0}$. Hence:

$$
\begin{gathered}
z^{ \pm}(x)=0 \text { for } x \geqq 2 u_{0}, \\
\left|z^{ \pm}(x+t)\right| \leqq c \sigma_{v}\left(\frac{x+t}{2}\right)+c \int_{x}^{2 u_{0}-x}\left|z^{ \pm}(u+t)\right| \sigma_{v}\left(\frac{x+u}{2}\right) d u .
\end{gathered}
$$

Using $(R)$, we are still led to the bound (5.25). Lastly, it is obvious that (5.25) holds in the case $\sigma_{v}(u) \equiv 0$. With the help of the bounds (4.23) and (5.25), it is easy to see from the formula (5.17) that $z^{ \pm}(x)$ is continuously differentiable; $s^{ \pm}(x)$ is therefore also continuously differentiable and as a consequence the formula (5.19) is justified. $z^{ \pm \prime}$ being given by the formula

$$
\begin{aligned}
& z^{ \pm \prime}(2 x)=-F^{ \pm}(x) F^{\mp \prime}(x) z^{ \pm}(2 x)+F^{ \pm}(x) A^{\mp}(x, x) z^{ \pm}(2 x) \\
& \quad+\left.F^{ \pm}(x) \frac{\partial}{\partial u} A^{ \pm}(u, x)\right|_{u=x}-F^{ \pm}(x) \int_{x}^{\infty} \frac{\partial}{\partial x} A^{\mp}(x, u) z^{ \pm}(u+z) d u, \quad x \geqq 0,
\end{aligned}
$$

it is not difficult to prove that it satisfies the bound

Clearly

$$
\left|z^{ \pm \prime}(2 x)\right| \leqq c\left[\sigma_{v}^{2}(x)+W(x)\right], \quad x \geqq 0 .
$$

hence:

$$
x \sigma_{v}(x) \leqq \int_{x}^{\infty} y\left[|U(y)|+\left|Q^{\prime}(y)\right|\right] d y<\infty, \quad x \geqq 0 ;
$$

$$
\int_{0}^{\infty} x \sigma_{v}^{2}(x) d x \leqq c \int_{0}^{\infty} \sigma_{v}(x) d x<\infty
$$


On the other hand, we see from ( $4.23 \mathrm{~b})$ that

$$
\int_{0}^{\infty} x W(x) d x<\infty
$$

As a consequence of (5.30), (5.31) and (5.32),

$$
\int_{0}^{\infty} x\left|z^{ \pm \prime}(x)\right| d x<\infty
$$

To recapitulate:

Theorem 5.1. If the conditions $I, I I, I I I$ and $I V$ hold, the functions $A^{+}(x, t), A^{-}(x, t), F^{+}(x)$ and $F^{-}(x)$, which generate the Jost solutions $f^{+}(k, x)$ and $f^{-}(k, x)$, satisfy the fundamental equations (5.17), (5.20) and (5.21). The function $z^{ \pm}(x)$ is continuously differentiable for $x \geqq 0$, satisfies the bound (5.25) and $x z^{ \pm \prime}(x)$ is integrable.

For real $U$ and $Q$, we easily obtain, in view of (3.20), (3.23), (3.22) and (3.18), the following conjugacy relations:

$$
\begin{gathered}
\overline{C_{n}^{ \pm}}=C_{n}^{\mp}, \\
\overline{p^{ \pm}(x)}=p^{\mp}(x), \\
\overline{s^{ \pm}(x)}=s^{\mp}(x), \\
\overline{z^{ \pm}(x)}=z^{\mp}(x) .
\end{gathered}
$$

\section{The Inverse Problem}

Our purpose, in this section, is to give a method of solving the inverse problem set in the introduction, i.e. of constructing the potentials $U(x)$ and $Q(x)$ from the knowledge of the binding energies $E_{n}^{+}$and of the scattering "matrix" $S^{+}(k)(k>0)$. Up to now, we have proved that, given potentials $U(x)$ and $Q(x)$ satisfying the Assumptions I, II, III and IV, the Jost solution $f^{ \pm}(k, x)$ is generated by two functions $A^{ \pm}(x, t)$ and $F^{ \pm}(x)$, where $\left(A^{+}(x, t), A^{-}(x, t), F^{+}(x), F^{-}(x)\right)$ is a solution of the system of fundamental equations (5.17), (5.20) and (5.21). These equations are determined from the data of the fundamental functions $z^{+}(x)$ and $z^{-}(x)(x \geqq 0)$. The starting point of our method is the investigation of this system. A complete solution of it is carried out only in some special cases at the end of the section. Nevertheless, for some sufficiently large values of the real positive number $\mathfrak{a}$, we solve, under not too restrictive conditions upon the input functions $z^{+}(x)$ and $z^{-}(x)$, the following 
system of equations:

$$
\begin{array}{cl}
A^{+}(x, t)=F^{-}(x) z^{+}(x+t)+\int_{x}^{\infty} A^{-}(x, u) z^{+}(u+t) d u, & t \geqq x \geqq \mathfrak{a}, \\
A^{-}(x, t)=F^{+}(x) z^{-}(x+t)+\int_{x}^{\infty} A^{+}(x, u) z^{-}(u+t) d u, & t \geqq x \geqq \mathfrak{a}, \\
F^{+}(x)-F^{-}(x)=\int_{x}^{\infty}\left[A^{-}(x, t)-A^{+}(x, t)\right] d t, \quad x \geqq \mathfrak{a}, \\
F^{+}(x) F^{-}(x)=1, \quad x \geqq \mathfrak{a}, \\
\lim _{x \rightarrow \infty} F^{ \pm}(x)=1, \quad x \geqq \mathfrak{a} ;
\end{array}
$$

if $a=0$, this system is identical with the system (5.17)-(5.20)-(5.21).

\section{§1. Solution of the System of Fundamental Equations (6.1), (6.2) and (6.3)}

We make the following assumption on the input functions $z^{+}(x)$ and $z^{-}(x)$ :

Assumption $I^{\prime}$. The function $z^{ \pm}(x)$ is continuous for $x \geqq 0$ and bounded by a function $\sigma_{0}\left(\frac{x}{2}\right)$, positive, non-increasing and integrable for $x \geqq 0$ :

$$
\begin{aligned}
& \left|z^{ \pm}(x)\right| \leqq \sigma_{0}\left(\frac{x}{2}\right), \\
& \int_{0}^{\infty} \sigma_{0}(x) d x<\infty .
\end{aligned}
$$

We seek the functions $A^{+}(x, t)$ and $A^{-}(x, t)$ belonging to the class $\mathfrak{r}_{\mathfrak{a}}{ }^{8}$ and the functions $F^{+}(x)$ and $F^{-}(x)$ continuous and bounded for $x \geqq \mathfrak{a}$, which verify the system of Eqs. (6.1), (6.2) and (6.3). We set

$$
R_{x}=\int_{2 x}^{\infty} \sigma_{0}\left(\frac{t}{2}\right) d t, \quad x \geqq 0 .
$$

Clearly, there exist $x_{0} \geqq 0$ and $x_{1} \geqq 0$ such that

$$
\begin{aligned}
& R_{x}<1 \text { for } x \geqq x_{0}, \\
& R_{x}<\frac{1}{2} \text { for } x \geqq x_{1} .
\end{aligned}
$$

We now prove that, for $t \geqq x \geqq x_{0}, A^{+}(x, t)$ and $A^{-}(x, t)$ are uniquely determined by $(6.1 \mathrm{a})$ and $(6.1 \mathrm{~b})$, given $F^{+}(x)$ and $F^{-}(x)$. For this, it is sufficient to show that, $x$ being fixed, the two following coupled Fredholm

${ }^{8}$ The class $\mathfrak{A}_{a}$ is defined as is the class $\mathfrak{A}$ in Section 4 , but for $t \geqq x \geqq \mathfrak{a}$ instead of $t \geqq x \geqq 0$. 
integral equations with kernels $z^{+}(u+t)$ and $z^{-}(u+t)$

$$
\begin{aligned}
& \Delta^{+}(x, t)=\int_{x}^{\infty} \Delta^{-}(x, u) z^{+}(u+t) d u, \quad t \geqq x \geqq x_{0}, \\
& \Delta^{-}(x, t)=\int_{x}^{\infty} \Delta^{+}(x, u) z^{-}(u+t) d u, \quad t \geqq x \geqq x_{0},
\end{aligned}
$$

have only the trivial solution. Inserting (6.7b) and (6.7a) in the right hand side of $(6.7 \mathrm{a})$ and $(6.7 \mathrm{~b})$ respectively, and interchanging the orders of integration we obtain

where

$$
\Delta^{ \pm}(x, t)=\int_{x}^{\infty} \Delta^{ \pm}(x, u) \mathfrak{E}_{x}^{\mp}(u, t) d u, \quad t \geqq x \geqq x_{0},
$$

$$
\begin{array}{r}
\mathfrak{E}_{x}^{ \pm}(u, t)=\mathfrak{E}_{x, 0}^{ \pm}(u, t)=\int_{x}^{\infty} z^{ \pm}(u+v) z^{\mp}(v+t) d v=\mathfrak{E}_{x}^{\mp}(t, u), \\
u \geqq x, \quad t \geqq x, \quad x \geqq 0 .
\end{array}
$$

Clearly, we have the following bounds for $u \geqq x, t \geqq x, x \geqq 0$ :

$$
\begin{aligned}
& \left|\mathfrak{E}_{x}^{ \pm}(u, t)\right| \leqq R_{x} \sigma_{0}\left(\frac{u+x}{2}\right), \\
& \left|\mathfrak{E}_{x}^{ \pm}(u, t)\right| \leqq R_{x} \sigma_{0}\left(\frac{t+x}{2}\right), \\
& \left|\mathfrak{E}_{x}^{ \pm}(u, t)\right| \leqq R_{x} \sigma_{0}^{1 / 2}\left(\frac{u+x}{2}\right) \sigma_{0}^{1 / 2}\left(\frac{t+x}{2}\right) .
\end{aligned}
$$

It is not difficult, from (6.8) and (6.10), to prove by induction that

$$
\Delta^{ \pm}(x, t)=\int_{x}^{\infty} \Delta^{ \pm}(x, u) \mathbb{E}_{x, n}^{\mp}(u, t) d u, \quad t \geqq x \geqq x_{0}, \quad n \geqq 0,
$$

where

$$
\begin{aligned}
\mathfrak{E}_{x, n}^{ \pm}(u, t) & =\int_{x}^{\infty} \mathfrak{E}_{x, k}^{ \pm}(u, v) \mathfrak{E}_{x, n-1-k}^{ \pm}(v, t) d v, \\
& =\mathfrak{E}_{x, n}^{\mp}(t, u), \quad u \geqq x, \quad t \geqq x, \quad 0 \leqq k \leqq n-1, \quad n \geqq 1,
\end{aligned}
$$

and

$$
\begin{aligned}
\left|\mathfrak{E}_{x, n}^{ \pm}(u, t)\right| \leqq R_{x}^{2 n+1} \sigma_{0}\left(\frac{u+x}{2}\right), & n \geqq 0, \\
\left|\mathfrak{E}_{x, n}^{ \pm}(u, t)\right| \leqq R_{x}^{2 n+1} \sigma_{0}\left(\frac{t+x}{2}\right), & n \geqq 0 .
\end{aligned}
$$


Hence, we can write from (6.11) and (6.13a)

where

$$
\left|\Delta^{ \pm}(x, t)\right| \leqq R_{x}^{2(n+1)} M, \quad t \geqq x \geqq x_{0}, \quad n \geqq 0,
$$

$$
M=\sup _{t \geqq x \geqq 0}\left|\Delta^{ \pm}(x, t)\right|,
$$

and this yields the desired result

$$
\Delta^{ \pm}(x, t)=0, \quad t \geqq x \geqq x_{0},
$$

because the limit of the right hand side is obviously zero as $n \rightarrow \infty$. Now, we apply the method of successive approximations to (6.1 a) and (6.1 b), considered as coupled Fredholm integral equations with kernels $z^{+}(u+t)$ and $z^{-}(u+t)$ and with solution $\left(A^{+}(x, t), A^{-}(x, t)\right)$. It is straightforward but tedious to show that the unique solution, for $t \geqq x \geqq x_{0}$, of the system (6.1 a) $-(6.1 \mathrm{~b})$ is given by

$$
A^{ \pm}(x, t)=F^{\mp}(x) \Psi_{x}^{ \pm}(x, t)+F^{ \pm}(x) \Phi_{x}^{\mp}(x, t), \quad t \geqq x \geqq x_{0},
$$

with

$$
\begin{aligned}
& \Psi_{x}^{ \pm}(x, t)=z^{ \pm}(x+t)+\int_{x}^{\infty} z^{ \pm}(x+u) \Phi_{x}^{\mp}(u, t) d u, \quad t \geqq x \geqq x_{0}, \\
& \Phi_{x}^{ \pm}(u, t)=\sum_{n=0}^{\infty} \mathfrak{E}_{x, n}^{ \pm}(u, t)=\Phi_{x}^{\mp}(t, u), \quad u \geqq x, \quad t \geqq x, \quad x \geqq x_{0} .
\end{aligned}
$$

The mainstay of the proof is the uniform convergence (because of the bound (6.13)) of the series (6.18) for $u \geqq x, t \geqq x, x \geqq x_{0}$. The following formulas are also needed:

$$
\begin{gathered}
\int_{x}^{\infty} z^{ \pm}(u+v) \mathfrak{E}_{x, n}^{ \pm}(w, v) d v=\int_{x}^{\infty} z^{ \pm}(w+v) \mathfrak{E}_{x, n}^{\mp}(v, u) d v, \\
n \geqq 0, \quad u \geqq x, \quad w \geqq x, \quad x \geqq 0, \\
\int_{x}^{\infty} z^{ \pm}(u+v) \Phi_{x}^{ \pm}(w, v) d v=\int_{x}^{\infty} z^{ \pm}(w+v) \Phi_{x}^{\mp}(v, u) d v, \\
u \geqq x, \quad w \geqq x, \quad x \geqq x_{0} .
\end{gathered}
$$

It is now easy to see that, in the system of fundamental equations, $(6.1 \mathrm{a})$ and $(6.1 \mathrm{~b})$ can be replaced by (6.16) for $t \geqq x \geqq x_{0}$. Furthermore, for $u \geqq x, t \geqq x, x \geqq x_{0}, \Phi_{x}^{ \pm}(u, t)$ is continuous with respect to $(x, u, t)$ and has the following bounds:

$$
\begin{aligned}
& \left|\Phi_{x}^{ \pm}(u, t)\right| \leqq \frac{R_{x}}{1-R_{x}^{2}} \sigma_{0}\left(\frac{u+x}{2}\right), \\
& \left|\Phi_{x}^{ \pm}(u, t)\right| \leqq \frac{R_{x}}{1-R_{x}^{2}} \sigma_{0}\left(\frac{t+x}{2}\right) ;
\end{aligned}
$$


for $t \geqq x \geqq x_{0}, \Psi_{x}^{ \pm}(x, t)$ is continuous with respect to $(x, t)$, and

$$
\left|\Psi_{x}^{ \pm}(x, t)\right| \leqq \frac{1}{1-R_{x}^{2}} \sigma_{0}\left(\frac{x+t}{2}\right) .
$$

Inserting (6.16) in (6.2), we obtain

$$
\begin{aligned}
& F^{+}(x) {\left[1-\int_{x}^{\infty}\left(\Psi_{x}^{-}(x, u)-\Phi_{x}^{-}(x, u)\right) d u\right] } \\
& \quad=F^{-}(x)\left[1-\int_{x}^{\infty}\left(\Psi_{x}^{+}(x, u)-\Phi_{x}^{+}(x, u)\right) d u\right], \quad x \geqq x_{0} .
\end{aligned}
$$

Since (6.21) and (6.22) together give:

$$
\left|\int_{x}^{\infty}\left(\Psi_{x}^{ \pm}(\mathrm{x}, \mathrm{u})-\Phi_{x}^{ \pm}(\mathrm{x}, \mathrm{u})\right) \mathrm{du}\right| \leqq \frac{\mathrm{R}_{x}}{1-R_{x}}, \quad \mathrm{x} \geqq \mathrm{x}_{0},
$$

we see, in view of (6.6b), that the second factor of each side of (6.23) does not vanish for $x \geqq x_{1}$. Then, recalling (6.3a), we obtain:

$$
\left[F^{ \pm}(x)\right]^{2}=\frac{1-\int_{x}^{\infty}\left(\Psi_{x}^{ \pm}(x, u)-\Phi_{x}^{ \pm}(x, u)\right) d u}{1-\int_{x}^{\infty}\left(\Psi_{x}^{\mp}(x, u)-\Phi_{x}^{\mp}(x, u)\right) d u}, \quad x \geqq x_{1} .
$$

$F^{ \pm}(x)$ is completely determined by the condition $(6.3 \mathrm{~b})$. Clearly, $F^{ \pm}(x)$ is continuous for $x \geqq x_{1}$ and has the bound:

$$
\left|F^{ \pm}(x)\right| \leqq \frac{1}{\left(1-2 R_{x}\right)^{\frac{1}{2}}}, \quad x \geqq x_{1} .
$$

Thus $A^{ \pm}(x, t)$ given by (6.16) is continuous for $t \geqq x \geqq x_{1}$ and, because of the bound

$$
\left|A^{ \pm}(x, t)\right| \leqq \frac{1}{\left(1-R_{x}\right)\left(1-2 R_{x}\right)^{\frac{1}{2}}} \sigma_{0}\left(\frac{x+t}{2}\right), \quad t \geqq x \geqq x_{1},
$$

$A^{ \pm}(x, t)$ belongs to the class $\mathfrak{A}_{x_{1}}$. So, we have proved that the system of fundamental Eqs. (6.1), (6.2) and (6.3) has a unique solution $\left(A^{+}(x, t)\right.$, $\left.A^{-}(x, t), F^{+}(x), F^{-}(x)\right)$ for $\mathfrak{a} \geqq x_{1}$.

\section{§ 2. Solution of the System of Equations (6.1) and (6.2)}

Now, we suppose $\mathfrak{a} \geqq x_{1}$. Let $a^{ \pm}(x, t)$ be a function belonging to the class $\mathfrak{A}_{a}$ and $f^{ \pm}(x)$ a function continuous and bounded for $x \geqq \mathfrak{a}$. If $\left(a^{+}(x, t), a^{-}(x, t), f^{+}(x), f^{-}(x)\right)$ is a solution of the system of Eqs. (6.1) 
and (6.2), i.e. if

$$
\begin{gathered}
a^{ \pm}(x, t)=f^{\mp}(x) z^{ \pm}(x+t)+\int_{x}^{\infty} a^{\mp}(x, u) z^{ \pm}(u+t) d u, \quad t \geqq x \geqq \mathfrak{a}, \\
f^{+}(x)-f^{-}(x)=\int_{x}^{\infty}\left[a^{-}(x, t)-a^{+}(x, t)\right] d t, \quad x \geqq \mathfrak{a},
\end{gathered}
$$

the results obtained in $\S 1$ are still valid up to and including (6.24) if $A^{ \pm}(x, t)$ is replaced by $a^{ \pm}(x, t), F^{ \pm}(x)$ by $f^{ \pm}(x), x_{0}$ and $x_{1}$ by $\mathfrak{a}$. This is true, in particular, for the formula (6.23) and hence

$$
f^{+}(x)=\frac{f^{-}(x)}{F^{-}(x)} F^{+}(x), \quad x \geqq \mathfrak{a} .
$$

Using (6.16), we conclude finally that there exists a unique function $U(x)$ defined and continuous for $x \geqq \mathfrak{a}$ such that

$$
\begin{gathered}
f^{ \pm}(x)=U(x) F^{ \pm}(x), \quad x \geqq \mathfrak{a}, \\
a^{ \pm}(x, t)=U(x) A^{ \pm}(x, t), \quad t \geqq x \geqq \mathfrak{a} .
\end{gathered}
$$

\section{§ 3. Properties of the Solution $\left(A^{+}(x, t), A^{-}(x, t), F^{+}(x), F^{-}(x)\right)$}

From here on, $\mathrm{I}^{\prime}$ is replaced by the stronger Assumption II':

Assumption $I I^{\prime} . z^{ \pm}(x)$ is twice continuously differentiable for $x \geqq 0$, and there exist functions $\sigma_{0}(x), \sigma_{1}(x)$ and $\sigma_{2}(x)$, positive and nonincreasing for $x \geqq 0$ such that

$$
\begin{array}{ll}
\left|z^{ \pm}(x)\right| \leqq \sigma_{0}\left(\frac{x}{2}\right) \text { and } & \int_{0}^{\infty} x \sigma_{0}(x) d x<\infty, \\
\left|z^{ \pm \prime}(x)\right| \leqq \sigma_{1}\left(\frac{x}{2}\right) \text { and } & \int_{0}^{\infty} x^{2} \sigma_{1}(x) d x<\infty, \\
\left|z^{ \pm \prime}(x)\right| \leqq \sigma_{2}\left(\frac{x}{2}\right) \text { and } & \int_{0}^{\infty} x^{2} \sigma_{2}(x) d x<\infty .
\end{array}
$$

We set

$$
\begin{aligned}
& \tilde{\sigma}_{0}(x)=\int_{2 x}^{\infty} \sigma_{1}\left(\frac{t}{2}\right) d t, \\
& \tilde{\sigma}_{1}(x)=\int_{2 x}^{\infty} \sigma_{2}\left(\frac{t}{2}\right) d t .
\end{aligned}
$$


Clearly, from Assumption II',

$$
\begin{aligned}
& \int_{0}^{\infty} x \tilde{\sigma}_{0}(x) d x<\infty, \\
& \int_{0}^{\infty} \mathrm{x} \tilde{\sigma}_{1}(x) d x<\infty .
\end{aligned}
$$

Let us also set

$$
S_{x}=\sup \left(\tilde{\sigma}_{0}(x), \sigma_{0}(x), R_{x}\right), \quad x \geqq 0 .
$$

There exists $x_{2} \geqq 0$ such that

We define $x_{3}$ by

$$
S_{x}<1 \text { for } x \geqq x_{2} .
$$

$$
x_{3}=\sup \left(x_{1}, x_{2}\right) .
$$

It is then straightforward but tedious to prove:

a) $F^{ \pm \prime}(x)$ exists and is continuous for $x \geqq x_{1}$, and

$$
\left|F^{ \pm \prime}(x)\right| \leqq C\left[\sigma_{0}(x)+\tilde{\sigma}_{0}(x)\right], \quad x \geqq x_{1} ;
$$

b) $F^{ \pm \prime \prime}(x)$ exists and is continuous for $x \geqq x_{3}$, and

$$
\left|F^{ \pm \prime \prime}(x)\right| \leqq C\left[\sigma_{0}(x)+\tilde{\sigma}_{0}(x)+\sigma_{1}(x)+\tilde{\sigma}_{1}(x)\right], \quad x \geqq x_{3} ;
$$

c) $\frac{\partial}{\partial x} A^{ \pm}(x, t)$ and $\frac{\partial}{\partial t} A^{ \pm}(x, t)$ exist and are continuous for $t \geqq x \geqq x_{1}$, and

$$
\begin{aligned}
& \left|\frac{\partial}{\partial x} A^{ \pm}(x, t)\right| \leqq C\left[\sigma_{0}\left(\frac{x+t}{2}\right)+\sigma_{1}\left(\frac{x+t}{2}\right)\right], \quad t \geqq x \geqq x_{1}, \\
& \left|\frac{\partial}{\partial t} A^{ \pm}(x, t)\right| \leqq C\left[\tilde{\sigma}_{0}\left(\frac{x+t}{2}\right)+\sigma_{1}\left(\frac{x+t}{2}\right)\right], \quad t \geqq x \geqq x_{1}, \\
& \left|\frac{d}{d x} A^{ \pm}(x, x)\right| \leqq C\left[\tilde{\sigma}_{0}(x)+\sigma_{1}(x)\right], \quad x \geqq x_{1} ;
\end{aligned}
$$

d) $\frac{\partial^{2} A^{ \pm}}{\partial x^{2}}(x, t)$ and $\frac{\partial^{2} A^{ \pm}}{\partial t^{2}}(x, t)$ exist and are continuous for $t \geqq x \geqq x_{3}$, and

$$
\begin{gathered}
\left|\frac{\partial^{2}}{\partial x^{2}} A^{ \pm}(x, t)\right| \leqq C\left[\sigma_{0}\left(\frac{x+t}{2}\right)+\sigma_{1}\left(\frac{x+t}{2}\right)+\sigma_{2}\left(\frac{x+t}{2}\right)\right], \quad t \geqq x \\
\left|\frac{\partial^{2}}{\partial t^{2}} A^{ \pm}(x, t)\right| \leqq C\left[\tilde{\sigma}_{1}\left(\frac{x+t}{2}\right)+\sigma_{2}\left(\frac{x+t}{2}\right)\right], \quad t \geqq x \geqq x_{3} .
\end{gathered}
$$


In Appendix $\mathrm{D}$, we give some bounds useful in deriving the preceding results. Note that if

a) $z^{ \pm}(x)$ is twice continuously differentiable for $x \geqq 0$,

b) $z^{ \pm}(\infty)=z^{ \pm \prime}(\infty)=z^{ \pm \prime \prime}(\infty)=0$

c) $z^{ \pm \prime}(x)$ is bounded by a positive and non-increasing function

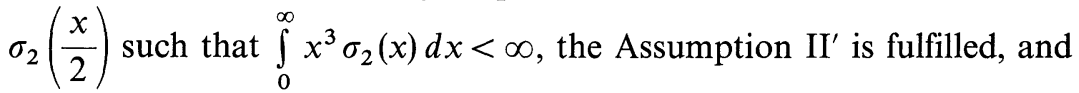
moreover, it is possible to choose $\sigma_{1}(x)$ and $\sigma_{0}(x)$ in such a way that $\tilde{\sigma}_{1} \equiv \sigma_{1}$ and $\tilde{\sigma}_{0} \equiv \sigma_{0}$. The bounds of $\S 3$ take then a simpler form.

\section{§ 4. Partial Differential Equations for $A^{+}(x, t), A^{-}(x, t), F^{+}(x)$ and $F^{-}(x)$}

Let us define the functions $Q(x), a^{ \pm}(x, t)$ and $f^{ \pm}(x)$ as follows:

$$
\begin{gathered}
Q(x)=\mp i \frac{F^{ \pm \prime}(x)}{F^{ \pm}(x)}, \quad x \geqq x_{3}, \\
a^{ \pm}(x, t)=\frac{\partial^{2}}{\partial x^{2}} A^{ \pm}(x, t)-\frac{\partial^{2}}{\partial t^{2}} A^{ \pm}(x, t) \pm 2 i Q(x) \frac{\partial}{\partial t} A^{ \pm}(x, t), \\
t \geqq x \geqq x_{3}, \\
f^{ \pm}(x)=F^{ \pm \prime}(x)-2 \frac{d}{d x} A^{ \pm}(x, x) \pm 2 i Q(x) A^{ \pm}(x, x), \quad x \geqq x_{3} .
\end{gathered}
$$

The above results show that $a^{ \pm}(x, t)$ belongs to the class $\mathfrak{A}_{x_{3}}$ and that $f^{ \pm}(x)$ is continuous and bounded for $x \geqq x_{3}$. We next apply the operator $\frac{\partial^{2}}{\partial x^{2}}-\frac{\partial^{2}}{\partial t^{2}} \pm 2 i Q(x) \frac{\partial}{\partial t}$ to both sides of (6.1); by means of differentiation under the integral sign and of integrations by parts, it is not difficult to prove that $\left(a^{+}(x, t), a^{-}(x, t), f^{+}(x), f^{-}(x)\right)$ is a solution of the equation (6.28) for $\mathfrak{a}=x_{3}$. Using the identity obtained by differentiating twice both sides of (6.2), we find that the Eq. (6.29) is also true for $\mathfrak{a}=x_{3}$. From the results in $\S 2$, we conclude that there exists a function $U(x)$ defined and continuous for $x \geqq x_{3}$ such that

$$
\begin{aligned}
& \left(\frac{\partial^{2}}{\partial x^{2}}-\frac{\partial^{2}}{\partial t^{2}} \pm 2 i Q(x) \frac{\partial}{\partial t}\right) A^{ \pm}(x, t)=U(x) A^{ \pm}(x, t), \quad t \geqq x \geqq x_{3}, \\
& F^{ \pm \prime \prime}(x)-2 \frac{d}{d x} A^{ \pm}(x, x) \pm 2 i Q(x) A^{ \pm}(x, x)=U(x) F^{ \pm}(x), \quad x \geqq x_{3} .
\end{aligned}
$$

It is easy to see from the formulas (6.45) and (6.49) with the help of the bounds established in $\S 3$, that $Q(x)$ is continuously differentiable for 
$x \geqq x_{3}, U(x)$ is continuous for $x \geqq x_{3}$ and that

$$
\begin{array}{ll}
|Q(x)| \leqq C\left[\sigma_{0}(x)+\tilde{\sigma}_{0}(x)\right], & x \geqq x_{3}, \\
\left|Q^{\prime}(x)\right| \leqq C\left[\sigma_{0}(x)+\tilde{\sigma}_{0}(x)+\sigma_{1}(x)+\tilde{\sigma}_{1}(x)\right], & x \geqq x_{3}, \\
|U(x)| \leqq C\left[\sigma_{0}(x)+\tilde{\sigma}_{0}(x)+\sigma_{1}(x)+\tilde{\sigma}_{1}(x)\right], & x \geqq x_{3} .
\end{array}
$$

Hence, using the Assumption II':

$$
\begin{aligned}
& \int_{x_{3}}^{\infty} x|Q(x)| d x<\infty, \\
& \int_{x_{3}}^{\infty} x\left|Q^{\prime}(x)\right| d x<\infty, \\
& \int_{x_{3}}^{\infty} x|U(x)| d x<\infty .
\end{aligned}
$$

\section{$\S 5$. Construction of $U$ and $Q$ from Given $z^{+}$and $z^{-}$}

Given functions $z^{+}(x)$ and $z^{-}(x)$ satisfying the Assumption $\mathrm{II}^{\prime}$, we propose to find potentials $U(x)$ and $Q(x)$, whose associated fundamental functions are precisely $z^{+}(x)$ and $z^{-}(x)$. The results obtained in $\S 4$ are not sufficient for our purpose. We shall assume that they hold, not only for $x \geqq x_{3}$ but also for $x \geqq 0$; this will be proved in some particular cases in $\S 7$. Then, from the important Theorem 4.2, we see that the Jost solution $f^{ \pm}(k, x)$ corresponding to the potentials $U(x)$ and $Q(x)$ defined by (6.49) and (6.45) is generated by the functions $A^{ \pm}(x, t)$ and $F^{ \pm}(x)$, themselves obtained from the data of $z^{+}$and $z^{-}$by solution of (6.1), (6.2) and (6.3) for $\mathfrak{a}=0$. Let $z_{v}^{+}(x)$ and $z_{v}^{-}(x)$ be the fundamental functions associated with the potentials $U(x)$ and $Q(x)$, which we also suppose satisfy the Assumptions III and IV. The generating functions $A^{ \pm}(x, t)$ and $F^{ \pm}(x)$ being unique for a given potential from Theorem 4.1, $z^{ \pm}(y)$ and $z_{v}^{ \pm}(y)$ are solutions of the same Volterra integral equation

$$
\begin{gathered}
z^{ \pm}(y)=F^{ \pm}\left(\frac{y}{2}\right) A^{ \pm}\left(\frac{y}{2}, \frac{y}{2}\right)-\int_{y}^{\infty} F^{ \pm}\left(\frac{y}{2}\right) A^{\mp}\left(\frac{y}{2}, v-\frac{y}{2}\right) z^{ \pm}(v) d v, \\
y \geqq 0,
\end{gathered}
$$

derived from (5.17) by setting $t=x, v=x+u, y=2 x$. Since $A^{ \pm}(x, t)$ belongs to the class $\mathfrak{A}$, and $F^{ \pm}(x), z^{ \pm}(x), z_{v}^{ \pm}(x)$ are bounded, it is easy to prove that

$$
z^{ \pm}(x) \equiv z_{v}^{ \pm}(x), \quad x \geqq 0 .
$$


Therefore, with the assumptions made, there is one pair of potentials $(U(x), Q(x))$ which reproduces the input functions $z^{+}(x)$ and $z^{-}(x)$ as fundamental functions. $U(x)$ and $Q(x)$ are given by (6.49) and (6.45). The pair $(U(x(, Q(x))$ is unique in the class $\mathfrak{C}$ of potentials satisfying the Assumptions I, II, III and IV since the system of fundamental equations has been proved to hold in these conditions.

\section{§ 6. Construction of $U$ and $Q$ from the Data of $E_{n}^{+}$and $S^{+}(k)(k>0)$}

Here, we suppose that the $N^{+}$binding energies $E_{n}^{+}$and the scattering matrix $S^{+}(k)(k>0)$ are exactly known from collision experiments, and we apply the results of $\S 5$ to construct the potentials $U(x)$ and $Q(x)$ which reproduce $E_{n}^{+}$and $S^{+}(k)(k>0)$. We assume we are given $N^{+}$numbers $C_{n}^{+}, N^{-}$numbers $C_{n}^{-}, N^{-}$numbers $E_{n}^{-}$and a function $S^{-}(k)(k>0)$; the quantities $C_{n}^{+}, N^{-}, C_{n}^{-}, E_{n}^{-}$and $S^{-}(k)(k>0)$ play the role of parameters, and may be chosen freely. We assume $N^{+}, N^{-}, E_{n}^{+}, E_{n}^{-}, C_{n}^{+}$, $C_{n}^{-}, S^{+}(k)$ and $S^{-}(k)(k>0)$, to be such that the input functions $z^{+}(x)$ and $z^{-}(x)(x>0)$ are determined via the formulas (5.14), (3.14), (3.13), (5.19) and (5.18), and that they verify the conditions of $\S 5$. We know then, from $\S 5$, how to construct the unique pair of potentials $(U(x), Q(x))$ belonging to $\mathfrak{C}$ which have $z^{+}(x)$ and $z^{-}(x)$ as associated fundamental functions. For $U(x)$ and $Q(x)$ to reproduce $E_{n}^{+}$and $S^{+}(k)(k>0)$, it is sufficient that the constructed function $F^{+}(x)$ satisfies the relation $(3: 13 \mathrm{~b})$, and that the following conditions be satisfied by the solution $\left(A^{+}(x, t)\right.$, $\left.A^{-}(x, t), F^{+}(x), F^{-}(x)\right)$ of the system of fundamental Eqs. (5.17), (5.20) and (5.21):

$$
\begin{gathered}
F^{+}(0) p^{-}(t)+\int_{0}^{\infty} A^{+}(0, u) p^{-}(u+t) d u=0, \quad t \geqq 0 \\
A^{-}(0, t)=F^{+}(0) s^{-}(t)+\int_{0}^{\infty} A^{+}(0, u) s^{-}(u+t) d u, \quad t \geqq 0, \\
-\left[F^{-}(0)\right]^{2} A^{+}(0,-t)=F^{+}(0) s^{-}(t)+\int_{0}^{\infty} A^{+}(0, u) s^{-}(u+t) d u, t \leqq 0
\end{gathered}
$$

(6.58), (6.59a) and (6.59b) are readily obtained by writing $f^{+}\left(k_{n}^{+}\right)=0$ and $f^{+}(k)=S^{+}(k) f^{-}(-k)(k \in \mathbb{R})$. Using the formula (5.17) for $x=0$, it is easy to see that either of the two formulas (6.58) and (6.59a) yields the other. Note that if the conditions (5.34), (3.22) and (3.21) hold, $U(x)$ and $Q(x)$ are real. If $S^{+}(k)=S^{-}(k)=S(k), C_{n}^{+}=C_{n}^{-}=C_{n}$ and $k_{n}^{+}=k_{n}^{-}=k_{n}$, then $Q=0$. 
An important question has still to be solved. One would like to know how far one can vary the input parameters $C_{n}^{+}, N^{-}, C_{n}^{-}, E_{n}^{-}$and $S^{-}(k)(k>0)$ and still retain the existence of a pair $(U(x), Q(x))$ solution of the inverse problem. This question has been already solved for real $U$ and $Q=0$ : we know [19] that, under very general conditions on $E_{n}$ and $S(k)$, the only restriction on the numbers $C_{n}$ for the inverse problem to be soluble is that they be positive. It should also be possible, by using similar arguments, to solve the question for real $U(x)$ and $Q(x)$. In general, we may expect many solutions to the problem.

\section{§7. Specific Examples}

In the real world $E_{n}^{+}$and $S^{+}(k)(k>0)$ must be found from collision experiments and $E_{n}^{-}, N^{-}, C_{n}^{+}, C_{n}^{-}, S^{-}(k)(k>0)$ are almost free parameters. In general they lead to complicated functions $z^{ \pm}$and so to complicated integral equations. Here we start from simple functions $z^{ \pm}$. In this way one can see how the method is applied without involving oneself in complicated numerical calculation.

First, we remark that there are cases in which our method of solution of the system of fundamental Eqs. (6.1), (6.2) and (6.3) holds for $a=0$. In fact, if we have

$$
R_{x=0}<\frac{1}{2}, \quad S_{x=0}<1,
$$

then the number $x_{3}$ may be chosen equal to zero in all the results of $\S 1,2,3,4$. If the conditions III and IV hold for the potentials $U(x)$ and $Q(x)$ that we construct, we also obtain the results of $\S 5$. This is the case if

$$
R_{x=0}<\frac{1}{5}
$$

since the Jost function $f^{ \pm}(k)$ constructed has then no zeros for $\operatorname{Im} k \leqq 0$.

We give now an exactly soluble example. We start from the following input functions:

$$
\begin{array}{ll}
z^{+}(t)=\alpha^{+} e^{-p t}, & t \geqq 0, \quad \alpha^{+} \in \mathbb{C}, \quad p>0, \\
z^{-}(t)=0, & t \geqq 0,
\end{array}
$$

and we suppose a) or b):
a) $\operatorname{Im} \alpha^{+} \neq 0$,
b) $\alpha^{+}$is real and $p>\alpha^{+}$. 
The system of fundamental Eqs. (5.17), (5.20) and (5.21) can be easily solved, with the solution

$$
\begin{aligned}
F^{ \pm}(x) & =\left(1-\frac{\alpha^{+}}{p} e^{-2 p x}\right)^{ \pm \frac{1}{2}}, \quad x \geqq 0, \\
A^{+}(x, t) & =\alpha^{+} e^{-p(x+t)} F^{-}(x), \quad t \geqq x \geqq 0, \\
A^{-}(x, t) & =0, \quad t \geqq x \geqq 0 ;
\end{aligned}
$$

Hence:

$$
\begin{aligned}
Q(x) & =-i \alpha^{+} e^{-2 p x}\left[F^{-}(x)\right]^{2}, \quad x \geqq 0, \\
U(x) & =\left(2 p \alpha^{+} e^{-2 p x}+\left(\alpha^{+}\right)^{2} e^{-4 p x}\right)\left[F^{-}(x)\right]^{4}, \quad x \geqq 0, \\
f^{-}(k, x) & =e^{-i k x} F^{-}(x), \quad x \geqq 0, \\
f^{+}(k, x) & =e^{-i k x} F^{-}(x)\left\{\left[F^{+}(x)\right]^{2} k-i p\right\}[k-i p]^{-1}, \quad x \geqq 0, \\
S^{+}(k) & =\left[S^{-}(-k)\right]^{-1}=\left[\left(1-\frac{\alpha^{+}}{p}\right) k-i p\right](k-i p)^{-1} .
\end{aligned}
$$

$f^{-}(k)$ never vanishes; $f^{+}(k)$ has a simple zero for $k=k_{0}, k_{0}$ being defined as

$$
k_{0}=i p\left(1-\frac{\alpha^{+}}{p}\right)^{-1}
$$

therefore, if $p>\operatorname{Re} \alpha^{+}$there is no bound state corresponding to $V^{+}$; if $\operatorname{Im} \alpha^{+} \neq 0$, and $p<\operatorname{Re} \alpha^{+}$, there is a bound state corresponding to $V^{+}$ and it is easily found that

$$
C_{0}^{-}=-\alpha^{+}\left(1-\frac{\alpha^{+}}{p}\right)^{-2}
$$

In both cases, it is easy to verify directly that the pair $(U(x), Q(x))$ is the only pair of potentials belonging to $\mathbb{C}$ which has the input functions $z^{+}(x)$ and $z^{-}(x)$ given by (6.62) as fundamental functions. Note that for real $\alpha^{+}, U(x)$ is real and $Q(x)$ is imaginary.

For appropriate values of the complex numbers $\alpha^{+}$and $\alpha^{-}$, we can, more generally, construct the unique pair of potentials $(U(x), Q(x))$ belonging to $\mathfrak{C}$ which has as fundamental functions

$$
z^{ \pm}(t)=\alpha^{ \pm} e^{-p t}, \quad t \geqq 0, \quad p>0 .
$$

We can always choose $\alpha^{+}$and $\alpha^{-}$in such a way that there is no bound 
state. We give the principal results:

$$
\begin{aligned}
& F^{ \pm}(x)=\left(1-\frac{\alpha^{ \pm}}{p} e^{-2 p x}+\frac{\alpha^{+} \alpha^{-}}{4 p^{2}} e^{-4 p x}\right)^{\frac{1}{2}} \\
& \cdot\left(1-\frac{\alpha^{\mp}}{p} e^{-2 p x}+\frac{\alpha^{+} \alpha^{-}}{4 p^{2}} e^{-4 p x}\right)^{-\frac{1}{2}}, \quad x \geqq 0, \\
& A^{ \pm}(x, t)=\left(1-\frac{\alpha^{+} \alpha^{-}}{4 p^{2}} e^{-4 p x}\right)^{-1} \\
& \cdot\left[F^{ \pm}(x) \frac{\alpha^{+} \alpha^{-}}{2 p} e^{-3 p x} e^{-p t}+F^{\mp}(x) \alpha^{ \pm} e^{-p x} e^{-p t}\right], \quad t \geqq x \geqq 0, \\
& Q(x)=\frac{-i\left(\alpha^{+}-\alpha^{-}\right) e^{-2 p x}\left(1-\frac{\alpha^{+} \alpha^{-}}{4 p^{2}} e^{-4 p x}\right)}{\left(1+\frac{\alpha^{+} \alpha^{-}}{4 p^{2}} e^{-4 p x}-\frac{\alpha^{-}}{p} e^{-2 p x}\right)\left(1+\frac{\alpha^{+} \alpha^{-}}{4 p^{2}} e^{-4 p x}-\frac{\alpha^{+}}{p} e^{-2 p x}\right)}, \\
& x \geqq 0 ;
\end{aligned}
$$

the form of $U(x)$ is more complicated ; let us only note that

$$
U(x) \underset{x \rightarrow \infty}{\sim} C e^{-2 p x} .
$$

Lastly, note that if $\alpha^{+}=\overline{\alpha^{-}}, U(x)$ and $Q(x)$ are real, and that if $\alpha^{+}=\alpha^{-}$, $Q(x)$ vanishes.

Acknowledgement. Professor P. C. Sabatier suggested this problem. We would like to thank him for many helpful discussions.

\section{Appendix A.1}

We want to estimate the behaviour of $\varphi^{ \pm}(k, x)$ for large values of $k$. For this, we start from the Neumann series (2.6) and write the general term as follows:

$$
\begin{aligned}
& \varphi_{n}^{ \pm}(k, x)=\alpha_{n}^{ \pm}(k, x)+\beta_{n}^{ \pm}(k, x)+\gamma_{n}^{ \pm}(k, x), \text { for } n \geqq 0, \\
& \gamma_{n}^{ \pm}(k, x)=a_{n}^{ \pm}(k, x)+b_{n}^{ \pm}(k, x)+c_{n}^{ \pm}(k, x), \text { for } n \geqq 0 \text {, }
\end{aligned}
$$

where:

$$
\begin{aligned}
& \alpha_{0}^{ \pm}(\mathrm{k}, \mathrm{x})=\frac{\mathrm{e}^{i k x}}{2 i k}, \quad \beta_{0}^{ \pm}(\mathrm{k}, \mathrm{x})=-\frac{\mathrm{e}^{-i k x}}{2 i k}, \\
& \gamma_{0}^{ \pm}(k, x)=a_{0}^{ \pm}(k, x)=b_{0}^{ \pm}(k, x)=c_{0}^{ \pm}(k, x)=0,
\end{aligned}
$$


and, for $n \geqq 1$ :

$$
\begin{aligned}
\alpha_{n}^{ \pm}(k, x)= & \pm \frac{e^{i k x}}{i} \int_{0}^{x} e^{-i k y} Q(y) \alpha_{n-1}^{ \pm}(k, y) d y, \\
\beta_{n}^{ \pm}(k, x)= & \mp \frac{e^{-i k x}}{i} \int_{0}^{x} e^{i k y} Q(y) \beta_{n-1}^{ \pm}(k, y) d y, \\
a_{n}^{ \pm}(k, x)= & \pm \frac{e^{i k x}}{i} \int_{0}^{x} e^{-i k y} Q(y) \beta_{n-1}^{ \pm}(k, y) d y \\
& \mp \frac{e^{-i k x}}{i} \int_{0}^{x} e^{i k y} Q(y) \alpha_{n-1}^{ \pm}(k, y) d y, \\
b_{n}^{ \pm}(k, x)= & \int_{0}^{x} \frac{\sin k(x-y)}{k} U(y)\left[\alpha_{n-1}^{ \pm}(k, y)+\beta_{n-1}^{ \pm}(k, y)\right] d y, \\
c_{n}^{ \pm}(k, x)= & \int_{0}^{x} \sin k(x-y)\left[\frac{U(y)}{k} \pm 2 Q(y)\right] \gamma_{n-1}^{ \pm}(k, y) d y
\end{aligned}
$$

We readily see that

$$
\begin{aligned}
& \alpha_{n}^{ \pm}(k, x)=\frac{e^{i k x}}{2 i k} \frac{\left(\mp i \int_{0}^{x} Q(y) d y\right)^{n}}{n !}, \quad \text { for } n \geqq 0, \\
& \beta_{n}^{ \pm}(k, x)=-\frac{e^{-i k x}}{2 i k} \frac{\left( \pm i \int_{0}^{x} Q(y) d y\right)^{n}}{n !}, \quad \text { for } n \geqq 0 .
\end{aligned}
$$

Hence:

$$
\begin{aligned}
& \sum_{n=0}^{\infty} \alpha_{n}^{ \pm}(k, x)=H^{ \pm}(x) \frac{e^{i k x}}{2 i k}, \\
& \sum_{n=0}^{\infty} \beta_{n}^{ \pm}(k, x)=-H^{\mp}(x) \frac{e^{-i k x}}{2 i k},
\end{aligned}
$$

where $H^{ \pm}(x)$ is given by $(2.8 \mathrm{~b})$. We are led to look for a bound for the quantity

$$
\varphi^{ \pm}(k, x)-\left(H^{ \pm}(x) \frac{e^{i k x}}{2 i k}-H^{\mp}(x) \frac{e^{-i k x}}{2 i k}\right), \text { i.e. for } \sum_{n=1}^{\infty} \gamma_{n}^{ \pm}(k, x) .
$$

It is straightforward to prove that

$$
\left|b_{n}^{ \pm}(k, x)\right| \leqq C \frac{e^{|b| x}}{|k|^{2}} \frac{(M(x))^{n}}{n !}, \text { for } n \geqq 1 \text { and }|k| \geqq 1,
$$

where $M(x)$ is defined by (2.8c). We obtain a similar bound for $a_{n}^{ \pm}(k, x)$ 
by integrating by parts each of the two terms of the expression (A.3c). We have for the first term, for $n \geqq 1$,

$$
\begin{aligned}
& \pm \frac{e^{i k x}}{i} \int_{0}^{x} e^{-i k y} Q(y) \beta_{n-1}^{ \pm}(k, y) d y \\
= & \frac{e^{i k x}}{i} \int_{0}^{x} \frac{e^{-2 i k y}}{2 i k} Q(y) \frac{\left( \pm i \int_{0}^{y} Q(t) d t\right)^{n-1}}{(n-1) !} d y \\
= & \frac{e^{i k x}}{i}\left[\frac{e^{-2 i k y}}{4 k^{2}} Q(y) \frac{\left( \pm i \int_{0}^{y} Q(t) d t\right)^{n-1}}{(n-1) !}\right]_{0}^{x} \\
& \pm \frac{e^{i k x}}{i} \int_{0}^{x} \frac{e^{-2 i k y}}{4 k^{2}} \\
& \cdot\left[Q^{\prime}(y) \frac{\left( \pm i \int_{0}^{y} Q(t) d t\right)^{n-1}}{(n-1) !}+Q^{2}(y)( \pm i)^{n-1} \frac{\left(\int_{0}^{y} Q(t) d t\right)^{n-2}}{(n-2) !}\right] d y,
\end{aligned}
$$

and an analogous result for the second term. We deduce from this:

$$
\begin{aligned}
\left|a_{n}^{ \pm}(k, x)\right| \leqq & C \frac{e^{|b| x}}{|k|^{2}}\left[\frac{(M(x))^{n-1}}{(n-1) !}+\frac{(M(x))^{n}}{n !}\right], \\
& \text { for } n \geqq 1 \text { and }|k| \geqq 1 .
\end{aligned}
$$

With the help of inequalities (A.6) and (A.8), it is not difficult to prove by induction the following bound for $\gamma_{n}^{+}(k, x)$ :

$$
\begin{aligned}
\left|\gamma_{n}^{ \pm}(k, x)\right| \leqq & C \frac{e^{|b| x}}{|k|^{2}}\left[\frac{(2 M(x))^{n-1}}{(n-1) !}+\frac{(2 M(x))^{n}}{n !}\right], \\
& \quad \text { for } n \geqq 1 \text { and }|k| \geqq 1,
\end{aligned}
$$

which leads to the inequality (2.8a).

\section{Appendix A.2}

Proceeding as in Appendix A.1, we obtain the behaviour of $f^{ \pm}(k, x)$ for large values of $k$. We write the integral Eq. (2.12) in the form

$$
\begin{aligned}
f^{ \pm}(k, x) e^{i k x}= & 1 \mp i \int_{x}^{\infty} Q(y) e^{i k y} f^{ \pm}(k, y) d y+\int_{x}^{\infty} \frac{U(y)}{2 i k} e^{i k y} f^{ \pm}(k, y) d y \\
& -\int_{x}^{\infty} \frac{e^{-2 i k(y-x)}}{2 i k}[U(y) \pm 2 k Q(y)] e^{i k y} f^{ \pm}(k, y) d y,
\end{aligned}
$$


and expand $f^{ \pm}(k, x) e^{i k x}$ as follows:

$$
\begin{aligned}
f^{ \pm}(k, x) e^{i k x} & =\sum_{n=0}^{\infty} g_{n}^{ \pm}(k, x), \\
g_{n}^{ \pm}(k, x) & =\mu_{n}^{ \pm}(k, x)+v_{n}^{ \pm}(k, x), \text { for } n \geqq 0, \\
v_{n}^{ \pm}(k, x) & =r_{n}^{ \pm}(k, x)+s_{n}^{ \pm}(k, x)+t_{n}^{ \pm}(k, x), \text { for } n \geqq 0,
\end{aligned}
$$

where:

$$
\mu_{0}^{ \pm}(k, x)=1, v_{0}^{ \pm}(k, x)=r_{0}^{ \pm}(k, x)=s_{0}^{ \pm}(k, x)=t_{0}^{ \pm}(k, x)=0,
$$

and, for $n \geqq 1$ :

$$
\begin{aligned}
& \mu_{n}^{ \pm}(k, x)=\mp i \int_{x}^{\infty} Q(y) \mu_{n-1}^{ \pm}(k, y) d y, \\
& r_{n}^{ \pm}(k, x)= \pm i \int_{x}^{\infty} e^{-2 i k(y-x)} Q(y) \mu_{n-1}^{ \pm}(k, y) d y, \\
& s_{n}^{ \pm}(k, x)=\frac{1}{2 i k} \int_{x}^{\infty} U(y)\left[1-e^{-2 i k(y-x)}\right] \mu_{n-1}^{ \pm}(k, y) d y, \\
& t_{n}^{ \pm}(k, x)=-i \int_{x}^{\infty}\left[\frac{U(y)}{2 k} \pm Q(y)\right]\left[1-e^{-2 i k(y-x)}\right] v_{n-1}^{ \pm}(k, y) d y .
\end{aligned}
$$

It is easy to see that

and

$$
\mu_{n}^{ \pm}(k, x)=\frac{\left(\mp i \int_{x}^{\infty} Q(y) d y\right)^{n}}{n !}, \text { for } n \geqq 0,
$$

$$
\sum_{n=0}^{\infty} \mu_{n}^{ \pm}(\mathrm{k}, \mathrm{x})=\mathrm{F}^{ \pm}(\mathrm{x})
$$

where $F^{ \pm}(x)$ is given by $(2.15 \mathrm{~b})$. We seek now a bound for the quantity $f^{ \pm}(k, x) e^{i k x}-F^{ \pm}(x)$, i.e. for $\sum_{n=1}^{\infty} v_{n}^{ \pm}(k, x)$. It is not difficult to obtain the inequality

$$
\left|s_{n}^{ \pm}(k, x)\right| \leqq \frac{C}{|k|} \frac{(P(x))^{n}}{n !}, \text { for } n \geqq 1 \text { and } k \neq 0,
$$

where $P(x)$ is defined by $(2.15 \mathrm{c})$. Integrating by parts (A.11f) and using the Assumption II, we derive also a bound for $r_{n}^{ \pm}(k, x)$ :

$$
\left|r_{n}^{+}(k, x)\right| \leqq \frac{C}{|k|}\left[\frac{(P(x))^{n-1}}{(n-1) !}+\frac{(P(x))^{n}}{n !}\right], \quad \text { for } n \geqq 1 \text { and } k \neq 0 \text {. }
$$


From formulas (A.13) and (A.14), we prove by induction that $\left|v_{n}^{ \pm}(k, x)\right| \leqq \frac{C}{|k|}\left[\frac{(2 P(x))^{n-1}}{(n-1) !}+\frac{(2 P(x))^{n}}{n !}\right]$, for $n \geqq 1$ and $|k| \geqq 1$.

Inequality (2.15a) follows immediately from (A.15).

\section{Appendix B}

We intend to prove the differentiability of $A^{ \pm}$with the help of the expansion (4.15). We note first that $\frac{\partial}{\partial x} A_{0}^{ \pm}(x, t)$ and $\frac{\partial}{\partial t} A_{0}^{ \pm}(x, t)$ exist and are continuous for $t \geqq x \geqq 0$, and are given by the formula

$$
\begin{aligned}
\frac{\partial}{\partial x} A_{0}^{ \pm}(x, t) & =\frac{\partial}{\partial t} A_{0}^{ \pm}(x, t) \\
& =-\frac{1}{4} F^{ \pm}\left(\frac{x+t}{2}\right)\left[U\left(\frac{x+t}{2}\right)+Q^{2}\left(\frac{x+t}{2}\right) \mp i Q\left(\frac{x+t}{2}\right)\right] .
\end{aligned}
$$

They also satisfy

$$
\left|\frac{\partial}{\partial x} A_{0}^{ \pm}(x, t)\right| \text { and }\left|\frac{\partial}{\partial t} A_{0}^{ \pm}(x, t)\right| \leqq m W\left(\frac{x+t}{2}\right)
$$

where $W(x)$ is defined by $(4.23 \mathrm{~b})$. Let us assume that the property $(P)$ :

$$
\text { “ } \frac{\partial}{\partial x} A_{n}^{ \pm}(x, t) \text { and } \frac{\partial}{\partial t} A_{n}^{ \pm}(x, t) \text { exist and are continuous for } t \geqq x \geqq 0 \text {, }
$$

and satisfy

$$
\begin{aligned}
& \left|\frac{\partial}{\partial x} A_{n}^{ \pm}(x, t)\right| \text { and }\left|\frac{\partial}{\partial t} A_{n}^{ \pm}(x, t)\right| \\
& \leqq m \sigma_{v}^{2}(x)\left[\left(2^{n}-1\right) \frac{(N(x))^{n-1}}{(n-1) !}+2^{n-3} \frac{(N(x))^{n}}{(n-1) !}\right]+m W\left(\frac{x+t}{2}\right) \frac{(N(x))^{n}}{n !}
\end{aligned}
$$

is true for a fixed arbitrary value of $n \geqq 1$. Using this and the results obtained for $A_{n}^{ \pm}(x, t)$ and $A_{n+1}^{ \pm}(x, t)$, it is tedious but straightforward to prove that $\frac{\partial}{\partial x} A_{n+1}^{ \pm}(x, t)$ and $\left.\frac{\partial}{\partial t} A_{n+1}^{ \pm}, x, t\right)$ exist and are continuous for 
$t \geqq x \geqq 0$ and are given by the following formulas:

$$
\begin{aligned}
\frac{\partial}{\partial x} A_{n+1}^{ \pm}(x, t)= & -\frac{1}{2} \int_{x}^{\infty} U(y) A_{n}^{ \pm}(y, t+y-x) d y \\
& -\frac{1}{2} \int_{x}^{\frac{x+t}{2}} U(y) A_{n}^{ \pm}(y, t+x-y) d y \\
& \pm \frac{i}{2} Q\left(\frac{x+t}{2}\right) A_{n}^{ \pm}\left(\frac{x+t}{2}, \frac{x+t}{2}\right) \\
& \pm i \int_{x}^{\infty} Q(y) \frac{\partial}{\partial t} A_{n}^{ \pm}(y, t+y-x) d y \\
& \pm i \int_{x}^{\frac{x+t}{2}} Q(y) \frac{\partial}{\partial t} A_{n}^{ \pm}(y, t+x-y) d y, \\
\frac{\partial}{\partial t} A_{n+1}^{ \pm}(x, t)= & +\frac{1}{2} \int_{x}^{\infty} U(y) A_{n}^{ \pm}(y, t+y-x) d y \\
& -\frac{1}{2} \int_{x}^{\frac{x+t}{2}} U(y) A_{n}^{ \pm}(y, t+x-y) d y \\
& \pm \frac{i}{2} Q\left(\frac{x+t}{2}\right) A_{n}^{ \pm}\left(\frac{x+t}{2}, \frac{x+t}{2}\right) \\
& \mp i \int_{x}^{\infty} Q(y) \frac{\partial}{\partial t} A_{n}^{ \pm}(y, t+y-x) d y \\
& \frac{x+t}{2}
\end{aligned}
$$

Inserting the bounds (B.3) and (4.14a) in the expressions (B.4) and (B.5), we show the validity of the bound (B.3) and hence of the property $(P)$ in the case $n+1$. As it is not difficult to prove $(P)$ for $n=1$, we conclude it holds for every $n \geqq 1$.

In view of (B.3), it is obvious that the series $\sum_{n=0}^{\infty} \frac{\partial}{\partial x} A_{n}^{ \pm}(x, t)$ and $\sum_{n=0}^{\infty} \frac{\partial}{\partial t} A_{n}^{ \pm}(x, t)$ converge uniformly on every compact set. Therefore, 
$\frac{\partial}{\partial x} A^{ \pm}(x, t)$ and $\frac{\partial}{\partial t} A^{ \pm}(x, t)$ exist and are continuous for $t \geqq x \geqq 0$ and,

$$
\begin{aligned}
& \frac{\partial}{\partial x} A^{ \pm}(x, t)=\sum_{n=0}^{\infty} \frac{\partial}{\partial x} A_{n}^{ \pm}(x, t), \\
& \frac{\partial}{\partial t} A^{ \pm}(x, t)=\sum_{n=0}^{\infty} \frac{\partial}{\partial t} A_{n}^{ \pm}(x, t) .
\end{aligned}
$$

The inequality $(4.23 \mathrm{a})$ follows immediately.

\section{Appendix C}

In this appendix, making the Assumptions I, II, III and IV, we prove a "completion formula".

To this purpose, we introduce the Green's function

$$
G^{ \pm}(k, x, y)= \begin{cases}\varphi^{ \pm}(k, x) \frac{f^{ \pm}(k, y)}{f^{ \pm}(k)}, & y \geqq x, \\ \varphi^{ \pm}(k, y) \frac{f^{ \pm}(k, x)}{f^{ \pm}(k)}, & 0 \leqq y \leqq x .\end{cases}
$$

Except for a finite number of non real poles corresponding to the bound states, $G^{ \pm}(k, x, y)$ is, for fixed $x$ and $y$, analytic in $k$ for $\operatorname{Im} k<0$ and continuous in $k$ for $\operatorname{Im} k \leqq 0$. Let $\psi(x)$ be a function twice differentiable and vanishing in the neighbourhoods of $x=0$ and $x=\infty$. We set

$$
\theta(x)=-\psi^{\prime \prime}(x)+U(x) \psi(x)
$$

It is easy to see that

$$
\psi(x)=\int_{0}^{\infty} G^{ \pm}(k, x, y)\left[\theta(y) \pm 2 k Q(y) \psi(y)-k^{2} \psi(y)\right] d y
$$

hence:

$$
\begin{aligned}
\frac{\psi(x)}{k}= & \frac{1}{k} \int_{0}^{\infty} G^{ \pm}(k, x, y) \theta(y) d y \pm 2 \int_{0}^{\infty} G^{ \pm}(k, x, y) Q(y) \psi(y) d y \\
& -k \int_{0}^{\infty} G^{ \pm}(k, x, y) \psi(y) d y
\end{aligned}
$$

We integrate both sides of (C.4) around a half-circle $|k|=R$ contained in the lower half of the complex $k$-plane and described in the positive sense. Thanks to the bounds (2.7) and (2.14), we see, using a Jordan's lemma, that the integral of the first term of the second side of (C.4) vanishes as $R \rightarrow \infty$. The same result holds for the integral of the second 
term; to prove this, we replace, in $G^{ \pm}(k, x, y), \varphi^{ \pm}(k, y)$ and $f^{ \pm}(k, y)$ by

$$
\begin{gathered}
\varphi^{ \pm}(k, y)=H^{ \pm}(y) \frac{e^{i k y}}{2 k}-H^{\mp}(y) \frac{e^{-i k y}}{2 i k}+R_{1}^{ \pm}(k, y), \\
f^{ \pm}(k, y)=F^{ \pm}(y) e^{-i k y}+R_{2}^{ \pm}(k, y),
\end{gathered}
$$

we integrate by parts with respect to $y$ the terms containing $e^{+i k y}$ and $e^{-i k y}$ in the expression of $\int_{0}^{\infty} G^{ \pm}(k, x, y) Q(y) \psi(y) d y$, and lastly, we make use of the bounds (2.7), (2.8), (2.14) and (2.15). The left hand side of (C.4) yields $i \pi \psi(x)$. We have therefore

$$
\psi(x)=-\frac{1}{i \pi} \lim _{R \rightarrow \infty} \int_{|k|=R} k d k \int_{0}^{\infty} G^{ \pm}(k, x, y) \psi(y) d y .
$$

Let us consider the same integral computed along a closed path, which is composed of the real segment $[-R,+R]$ and the half circle $|k|=R$ contained in the lower half of the complex $k$-plane; applying the theorem of residues, and making use of the formula (3.6) in the case $k=k_{n}^{ \pm}$, we obtain as $R \rightarrow \infty$

where

$$
\begin{aligned}
\psi(x)= & -\frac{1}{i \pi} \int_{0}^{\infty} k d k \int_{0}^{\infty}\left[G^{ \pm}(k, x, y)-G^{ \pm}(-k, x, y)\right] \psi(y) d y \\
& +\sum_{n=1}^{N \pm} B_{n}^{ \pm} \varphi^{ \pm}\left(k_{n}^{ \pm}, x\right) \int_{0}^{\infty} \varphi^{ \pm}\left(k_{n}^{ \pm}, y\right) \psi(y) d y,
\end{aligned}
$$

$$
B_{n}^{ \pm}=\frac{4 i\left(k_{n}^{ \pm}\right)^{2}}{\left.g^{ \pm}\left(k_{n}^{ \pm}\right) \frac{d}{d k} f^{ \pm}(k)\right|_{k=k_{n}^{ \pm}}} .
$$

We add the formula (C.8) corresponding to the index "+" and the formula (C.8) corresponding to the index "-"; then, with the help of the relations (2.9) and (3.7), we derive the following "completion formula" valid in the space of the functions $\psi(x)$ previously defined:

$$
\begin{aligned}
\psi(x)= & \int_{0}^{\infty} \varphi^{+}(k, x) d \varrho^{+}(k) \int_{0}^{\infty} \varphi^{+}(k, y) \psi(y) d y \\
& +\int_{0}^{\infty} \varphi^{-}(k, x) d \varrho^{-}(k) \int_{0}^{\infty} \varphi^{-}(k, y) \psi(y) d y \\
& +\sum_{n=1}^{N^{+}} \frac{\mathrm{B}_{n}^{+}}{2} \varphi^{+}\left(k_{n}^{+}, x\right) \int_{0}^{\infty} \varphi^{+}\left(k_{n}^{+}, y\right) \psi(y) d y \\
& +\sum_{n=1}^{N^{-}} \frac{B_{n}^{-}}{2} \varphi_{n}^{-}\left(k_{n}^{-}, x\right) \int_{0}^{\infty} \varphi^{-}\left(k_{n}^{-}, y\right) \psi(y) d y,
\end{aligned}
$$


where

$$
\frac{d \varrho^{ \pm}(k)}{d k}=\frac{1}{\pi} \frac{k^{2}}{f^{ \pm}(k) f^{\mp}(-k)}, \quad k \geqq 0 .
$$

Note that the coupled integral equations (5.17), which are of paramount importance in the study of the inverse problem, may be easily derived, in a purely formal sense, from the formulas (C.8).

\section{Appendix D}

In this appendix, we list some bounds useful in deriving the results of $\S 3$. These bounds are obtained without difficulty; we omit proofs. For $n \geqq 0, u \geqq x, t \geqq x, x \geqq 0$, we have:

$$
\begin{aligned}
& \left|\frac{\partial}{\partial x} \mathfrak{E}_{x, n}^{ \pm}(u, t)\right| \leqq(2 n+1) R_{x}^{2 n} \sigma_{0}\left(\frac{x+u}{2}\right) \sigma_{0}\left(\frac{x+t}{2}\right), \\
& \left|\frac{\partial}{\partial u} \mathfrak{E}_{x, n}^{ \pm}(u, t)\right| \leqq R_{x}^{2 n} \sigma_{0}\left(\frac{t+x}{2}\right) \tilde{\sigma}_{0}\left(\frac{x+u}{2}\right) ;
\end{aligned}
$$

for $u \geqq x, t \geqq x, x \geqq x_{0}$ :

$$
\begin{gathered}
\left|\frac{\partial}{\partial x} \Phi_{x}^{ \pm}(u, t)\right| \leqq \frac{1+R_{x}^{2}}{\left(1-R_{x}^{2}\right)^{2}} \sigma_{0}\left(\frac{x+u}{2}\right) \sigma_{0}\left(\frac{x+t}{2}\right), \\
\left|\frac{\partial}{\partial u} \Phi_{x}^{ \pm}(t, u)\right| \text { and }\left|\frac{\partial}{\partial u} \Phi_{x}^{ \pm}(u, t)\right| \leqq \frac{1}{1-R_{x}^{2}} \sigma_{0}\left(\frac{x+t}{2}\right) \tilde{\sigma}_{0}\left(\frac{x+u}{2}\right) ;
\end{gathered}
$$

for $t \geqq x \geqq x_{0}$ :

$$
\begin{gathered}
\left|\frac{\partial}{\partial x} \Phi_{x}^{ \pm}(x, t)\right| \leqq C \sigma_{0}\left(\frac{x+t}{2}\right)\left[\sigma_{0}(x)+\tilde{\sigma}_{0}(x)\right] \\
\left|\frac{\partial}{\partial x} \Psi_{x}^{ \pm}(x, t)\right| \leqq C\left[\sigma_{0}(x) \sigma_{0}\left(\frac{x+t}{2}\right)+\tilde{\sigma}_{0}(x) \sigma_{0}\left(\frac{x+t}{2}\right)+\sigma_{1}\left(\frac{x+t}{2}\right)\right] \\
\left|\frac{\partial}{\partial t} \Psi_{x}^{ \pm}(x, t)\right| \leqq C\left[\sigma_{0}(x) \tilde{\sigma}_{0}\left(\frac{x+t}{2}\right)+\sigma_{1}\left(\frac{x+t}{2}\right)\right]
\end{gathered}
$$

for $u \geqq x, t \geqq x, x \geqq 0$ :

$\left|\frac{\partial^{2}}{\partial x^{2}} \mathfrak{E}_{x, 0}^{ \pm}(u, t)\right| \leqq C\left[\sigma_{0}(x) \sigma_{1}\left(\frac{x+t}{2}\right)+\sigma_{1}\left(\frac{x+u}{2}\right) \sigma_{0}\left(\frac{x+t}{2}\right)\right]$, 


$$
\begin{aligned}
& \left|\frac{\partial^{2}}{\partial x^{2}} \mathfrak{E}_{x, n}^{ \pm}(u, t)\right| \leqq(4(n-1) n+10 n) S_{x}^{2 n} \sigma_{0}(x) \sigma_{0}\left(\frac{x+t}{2}\right) \\
& +S_{x}^{2 n} \sigma_{0}\left(\frac{x+t}{2}\right) \sigma_{1}\left(\frac{x+u}{2}\right)+S_{x}^{2 n} \sigma_{0}(x) \sigma_{1}\left(\frac{x+t}{2}\right), n \geqq 1, \\
& \left|\frac{\partial^{2}}{\partial x \partial u} \mathfrak{E}_{x, 0}^{ \pm}(u, t)\right| \leqq \sigma_{0}\left(\frac{x+t}{2}\right) \sigma_{1}\left(\frac{x+u}{2}\right), \\
& \left|\frac{\partial^{2}}{\partial x \partial u} \mathfrak{E}_{x, n}^{ \pm}(u, t)\right| \leqq 2 n S_{x}^{2 n} \sigma_{0}(x) \sigma_{0}\left(\frac{x+t}{2}\right)+S_{x}^{2 n} \sigma_{0}\left(\frac{x+t}{2}\right) \sigma_{1}\left(\frac{x+u}{2}\right) \text {, } \\
& n \geqq 1 \text {, } \\
& \left|\frac{\partial^{2}}{\partial u^{2}} \mathfrak{E}_{x, n}^{ \pm}(u, t)\right| \leqq R_{x}^{2 n} \sigma_{0}\left(\frac{x+t}{2}\right) \tilde{\sigma}_{1}\left(\frac{x+u}{2}\right), \quad n \geqq 0 \text {; }
\end{aligned}
$$

for $u \geqq x, t \geqq x, x \geqq x_{2}$ :

$$
\begin{aligned}
\left|\frac{\partial^{2}}{\partial x^{2}} \Phi_{x}^{ \pm}(u, t)\right| \leqq C\left[\sigma_{0}(x) \sigma_{0}\left(\frac{x+t}{2}\right)\right. & +\sigma_{0}\left(\frac{x+t}{2}\right) \sigma_{1}\left(\frac{x+u}{2}\right) \\
& \left.+\sigma_{0}(x) \sigma_{1}\left(\frac{x+t}{2}\right)\right]
\end{aligned}
$$

for $u \geqq x, t \geqq x, x \geqq x_{0}$ :

$$
\begin{aligned}
& \left|\frac{\partial^{2}}{\partial x \partial u} \Phi_{x}^{ \pm}(u, t)\right| \leqq C\left[\sigma_{0}(x) \sigma_{0}\left(\frac{x+t}{2}\right)+\sigma_{0}\left(\frac{x+t}{2}\right) \sigma_{1}\left(\frac{x+u}{2}\right)\right], \\
& \left|\frac{\partial^{2}}{\partial u^{2}} \Phi_{x}^{ \pm}(u, t)\right| \text { and }\left|\frac{\partial^{2}}{\partial u^{2}} \Phi_{x}^{ \pm}(t, u)\right| \leqq C \sigma_{0}\left(\frac{x+t}{2}\right) \tilde{\sigma}_{1}\left(\frac{x+u}{2}\right)
\end{aligned}
$$

for $t \geqq x \geqq x_{2}$ :

$$
\begin{aligned}
&\left|\frac{\partial^{2}}{\partial x^{2}} \Phi_{x}^{ \pm}(x, t)\right| \leqq C \sigma_{0}\left(\frac{x+t}{2}\right)\left[\sigma_{0}(x)+\sigma_{1}(x)+\tilde{\sigma}_{1}(x)\right] \\
&\left|\frac{\partial^{2}}{\partial x^{2}} \Psi_{x}^{ \pm}(x, t)\right| \leqq C\left[\sigma_{0}(x) \sigma_{1}\left(\frac{x+t}{2}\right)+\sigma_{2}\left(\frac{x+t}{2}\right)\right. \\
&\left.+\sigma_{0}\left(\frac{\mathrm{x}+\mathrm{t}}{2}\right)\left(\sigma_{0}(\mathrm{x})+\sigma_{1}(\mathrm{x})+\tilde{\sigma}_{1}(\mathrm{x})\right)\right] \\
&\left|\frac{\partial^{2}}{\partial t^{2}} \Psi_{x}^{ \pm}(x, t)\right| \leqq \sigma_{0}(x) \tilde{\sigma}_{1}\left(\frac{x+t}{2}\right)+\sigma_{2}\left(\frac{x+t}{2}\right)
\end{aligned}
$$




\title{
References
}

1. For a survey of this inverse problem, see Faddeyev, L.D.: The inverse problem in the quantum theory of scattering. J. Math. Phys. 4, 72 (1963).

Newton, R.G.: Scattering theory of waves and particles, Chapter 20. New York: Mc Graw-Hill Book Company, 1966.

2. Gel'fand, I. M., Levitan, B. M.: On the determination of a differential equation from its spectral function. Izvest. Akad. Nauk S.S.S.R. 15, 309 (1951); translated in Am. Math. Soc. Transl. 1, 253 (1955).

3. For the Marchenko method, see the book of Agranovich,Z.S., Marchenko, V.A.: The inverse problem of scattering theory. New York: Gordon and Breach 1963.

4. In the case of a complex energy-independent potential, this assumption has already been used by Gasymov, M. G.: Doklad. Akad. Nauk S.S.S.R. 165, 261 (1965); see also Bertero, M., Dillon, G.: An outline of scattering theory for absorptive potentials. Nuovo Cimento 2 A, 1024 (1971).

5. The first paper on the subject is that of Corinaldesi,E.: Construction of potentials from phase shift and binding energies of relativistic equations. Nuovo Cimento 11, 468 (1954). See also a recent paper of Degasperis, A.: On the inverse problem for the Klein-Gordon s-wave equation. J. Math. Phys. 11, 551 (1970), in which other references are given.

6. Sabatier,P.C.: Approach to scattering problems through interpolation formulas and application to spin-orbit potentials. J. Math. Phys. 9, 1241 (1968).

7. See, for example, De Alfaro, V., Regge, T.: Potential scattering, Chapter 3. Amsterdam: North Holland Publ. Comp. 1965.

8. See Chapter I, § 2, of Ref. [3].

9. For the case $Q=0$, see Chapter 4 of Ref. [7].

10. See Chapter I, $\S 4$, of Ref. [3].

11. See the paper of Bertero, M., Dillon, G.: Quoted in Ref. [4], formula (3.6).

12. Titchmarsh, E. C.: Introduction to the theory of Fourier integrals, p. 128. Oxford 1937.

13. See Chapter $1, \S 3$, of Ref. [3].

14. See, for example, Bochner, S., Chandrasekharan, K.: Fourier transforms, Annals of Mathematics Studies, Princeton University Press (1960), Theorem 60.

15. See Paley, R., Wiener, N.: Am. Math. Soc. Coll. Publ., XIX (1934), p. 63.

16. See Chapter III of Ref. [3].

17. See formulas (3.5), (3.7) and (3.8) of Ref. [6].

18. For the proof, see Ref. [3], Chapter III, Lemma 3.2.1.

19. See the paper of Faddeyev, L. D.: Quoted in Ref. [1], Theorem 12.1.

\author{
M. Jaulent \\ Département de Physique Mathématique \\ Faculté des Sciences \\ F-34 Montpellier, France
}

Note Added in Proof. After the completion of this work we read a paper by H. Cornille [J. Math. Phys. 11, $79(1970)$ ] in which he studied the reconstruction of $U(x)$ and $Q(x)$ from the $S$-matrix discontinuities in the complex $k$-plane for the Schrödinger equation (1.8a) with the energy-dependent potential $V^{ \pm}(k, x)=U(x) \pm 2\left(k^{2}+m^{2}\right)^{\frac{1}{2}} Q(x), m \geq 0$, in the case where $U(x)$ and $Q(x)$ are superpositions of exponential-type potentials - with the additional condition $U(x)=-Q^{2}(x)$, this equation reduces to the Klein-Gordon equation with the static potential $Q(x)$ for a particle (antiparticle) of mass $m$ and of energy $k-$. In this study he gave an extension of the Marchenko formalism: he showed that the Jost solution 
$f^{ \pm}(k, x)$ may be written in the form (Cornille formula 33)

$$
f^{ \pm}(k, x)=e^{-i k x} \cos \int_{x}^{\infty} Q(y) d y+\int_{x}^{\infty}\left[K_{1}(x, t) \pm\left(k^{2}+m^{2}\right)^{\frac{1}{2}} K_{2}(x, t)\right] e^{-i k t} d t,
$$

and by using dispersive methods he derived two coupled integral equations (Cornille formula 34) connecting $K_{1}(x, t)$ and $K_{2}(x, t)$ with certain functions easily deduced from the $S$-matrix discontinuities in the complex $k$-plane. Using the theorem of residues in the complex $k$-plane, it is easy to see that these functions are also easily deduced from the $S$-matrix for real $k$, from the binding energies and from certain other numbers associated with the bound states, and therefore are similar to our functions $z^{+}$and $z^{-}$. It is certainly possible to prove that this formalism is valid for a larger class of potentials $U(x)$ and $Q(x)$ though one cannot expect to use dispersive methods in general for the proof. However, for $m=0$, because of our assumption that $Q(x)$ is differentiable - which allowed us to perform useful integration by parts all along our paper - our formalism is not identical with the Cornille formalism. The connection between the two formalisms is nevertheless easy to do. Integrating by parts the second term in the integral of (i) and taking into account the relation $K_{2}(x, x)=\sin \int_{x}^{\infty} Q(t) d t$ (Cornille formula 39a), we find again our relation (4.20) if we set

$$
A^{ \pm}(x, t)=K_{1}(x, t) \mp i \frac{\partial}{\partial t} K_{2}(x, t) .
$$

Furthermore, starting from the Cornille integral equations for $K_{1}(x, t)$ and $K_{2}(x, t)$, and integrating by parts certain terms, we find, after some work, our integral Eqs. (5.17) for $A^{+}(x, t)$ and $A^{-}(x, t)$, but valid only in the case of superpositions of exponential-type potentials. 